\title{
Analysis of Budget for Interdiction on Multicommodity Network Flows
}

\author{
Pengfei Zhang · Neng Fan
}

December 28, 2015

\begin{abstract}
In this paper, we concentrate on computing several critical budgets for interdiction of the multicommodity network flows, and studying the interdiction effects of the changes on budget. More specifically, we first propose general interdiction models of the multicommodity flow problem, with consideration of both node and arc removals and decrease of their capacities. Then, to perform the vulnerability analysis of networks, we define the function $F(R)$ as the minimum amount of unsatisfied demands in the resulted network after worst-case interdiction with budget $R$. Specifically, we study the properties of function $F(R)$, and find the critical budget values, such as $R_{a}$, the largest value under which all demands can still be satisfied in the resulted network even under the worst-case interdiction, and $R_{b}$, the least value under which the worst-case interdiction can make none of the demands be satisfied. We prove that the critical budget $R_{b}$ for completely destroying the network is not related to arc or node capacities, and supply or demand amounts, but it is related to the network topology, the sets of source and destination nodes, and interdiction costs on each node and arc. We also observe that the critical budget $R_{a}$ is related to all of these parameters of the network. Additionally, we present formulations to estimate both $R_{a}$ and $R_{b}$. For the effects of budget increasing, we present the conditions under which there would be extra capabilities to interdict more arcs or nodes with increased budget, and also under which the increased budget has no effects for the interdictor. To verify these results and conclusions, numerical experiments on 12 networks with different numbers of commodities are performed.
\end{abstract}

Keywords Multicommodity flow $\cdot$ Network vulnerability $\cdot$ Interdiction · Critical budget

P. Zhang $\cdot$ N. Fan $(\bowtie)$

Department of Systems and Industrial Engineering, University of Arizona, Tucson, AZ 85721, USA.

E-mail: \{pfzhang,nfan\}@email.arizona.edu 


\section{Introduction}

The network interdiction problem, which aims to optimally impede network operations through limited disruption actions, was initially studied for military applications [1], such as delaying, disrupting or destroying enemy's supplies with limited resources (or budget). Recently, this problem has been studied and applied in many areas, such as reducing congestion in traffic network [2], controlling of infectious disease [3], intercepting of smuggling illegal items, for example, drugs [4], nuclear materials [5,6], etc., and many other applications, as discussed in [7].

This problem has been extensively studied since 1960s. Collado and Papp [7] summaried a large variety of models and algorithms. Models for this problem can be classified into several categories: interdiction on directed or undirected network, deterministic or stochastic interdiction, arc or node interdiction, etc. In some models, the network failures are given in a set. For example, Garg and Smith [8] studied a minimum cost survivable network design given a random set of scenarios each consisting of the simultaneous failures of some arcs. Liberatore et al. [9] presented a facility protection problem which is to optimally allocate resources to minimize the worst impact by random possible losses. Johnston et al. [10] considered a network protecting problem against random arc failures.

However, in most models, the failure scenario is determined by interdictor. Specifically, it is assumed that the interdictor chooses to make the maximum damage to the network. Israeli and Wood [11] studied the shortest path problem in which an interdictor tries to maximize the shortest $s$ - $t$ path length by interdicting the arcs or increasing the arc length. Golden [12] examined a similar problem but with a length constraint. Zenklusen [13] considered a matching interdiction problem, in which the interdictor tries to minimize the maximum weight of matchings by removing edges by limited budget. McMasters and Mustin [14] studied a military problem, in which the strike planner tries to find a most successful way to interdict the supply line of an enemy with a limited number of aircraft. Ghare et al. [15] described a similar problem in which a commander determines a best policy so that the resulted maximum flow can be minimized. Cormican et al. [16] proposed a stochastic version of the network interdiction problem where the interdictions are binary. Washburn and Wood [17] considered a network interdiction problem, in which the leader tries to maximize the probability of detecting the followers' travel. Fulkerson and Harding [18] considered the maximum shortest path problem and reformulated it to a minimum-cost network flow form. Recently, a stochastic minimum cost flow with arc failure uncertainty was also considered [19].

Aside from the above network problems, one category of models for interdiction is concerned with commodity flow problems. Such model involves combinatorial optimization, stochastic programming and game theory. Phillips [20] computed a optimal fixed-charge attack strategy to inhibit the capacity of a network to transport a commodity. Royset and Wood [21] considered a maximum-flow network-interdiction problem with respect to two objectives. Wollmer [2] studied a maximum flow network interdiction problem with arc removals. Wood [22] analyzed the maximum flow problem in a capacitated network, in which an interdictor tries to find the best way to minimize the maximum flow by interdicting arcs. Atkinson et al. [23] examined an 
optimal stopping problem to place sensors to stop a terrorist from driving a radiological weapon to a city. Lim and Smith [24] studied a network interdiction problem on a multicommodity flow network for both discrete and continuous interdictions. Smith et al. [25] examined a survivable network design problem in which the designer tries to fortify a network to defend against enemy attacks in various scenarios in the form of a three-level two-player game. Recently, Sullivan and Smith [26] studied interdiction problem in networks that reside in Euclidean space.

To solve multicommodity network flow interdiction problems, many algorithms have been developed such as dual-ascent heuristic procedure in [27] and primaldual algorithm in [28]. Royset and Wood [29] proposed an algorithm through a sequence of single-objective problems solved using Lagrangian relaxation and a specialized branch-and-bound algorithm. Kennington [30] surveyed some early algorithms. Wood [31] described basic theoretical models and solution methods for bilevel network interdiction model. For binary interdiction, Wood [22], Israeli and Wood [11] formulated the bilevel problem into a mixed-integer program (MIP) and then solved it directly. Lim and Smith [24] studied a linearized model and compared it with a penalty model that does not require linearization. For continuous case, Lim and Smith [24] proposed a partitioning algorithm with a heuristic procedure to estimate the optimal solution. Smith et al. [25] described a cutting-plane algorithm to obtain the optimal interdiction. This cutting-plane algorithm requires a large amount of computational time because many mixed-integer subproblems need to be solved and the number of subproblems is the number of all arcs of the network.

However, in these literatures, the budget for interdiction is always assumed to be a fixed value, and the effects of interdiction under different budgets are not analyzed. In most of these bilevel network interdiction models, it is always that the second level after the worst-case interdiction is still feasible, and duality and linearization techniques are general methods used for solving these bilevel programming problems. However, when budget increases to certain amount, the interdiction may cause that there is no feasible solution in the second level. For example, in the interdiction model of multicommodity flow, if the interdiction budget is large enough, there will be no feasible flow to satisfy all demands in the resulted network.

In this paper, we concentrate on computing several critical budgets for interdiction on the multicommodity network flows, and studying the interdiction effects of the changes of budget. The results from our study will help interdictor to decide the amount of limited resources, and how and where to perform interdiction on networks for expected destroy effects. More specifically, we first propose general interdiction models of the multicommodity flow problem, with consideration of both node and arc removals and decrease of their capacities. These models are formulated as bilevel programs, with cases for continuous and binary interdictions. To solve these programs, we reformulate them as mixed integer linear programs. In order to analyze the vulnerability of networks, we define the function $F(R)$ as the minimum amount of unsatisfied demands in the resulted network after worst-case interdiction with budget $R$, and perform the interdiction analysis regarding different values of budgets. Specifically, we study the properties of function $F(R)$, and find the critical budget values. For example, we obtain the value $R_{a}$, which is the largest value under which all demands can still be satisfied in the resulted network even under the worst-case 
interdiction, and $R_{b}$ the least value under which the worst-case interdiction can make none of the demands be satisfied. That is, for $0 \leq R \leq R_{a}, F(R)=0$, all demands will be satisfied; for $R_{a}<R<R_{b}$, only part of the demands can be satisfied; for $R \geq R_{b}$, $F(R)$ is equal to total demands and no demand can be satisfied. We prove that the critical budget $R_{b}$ for completely destroying the network is not related to arc or node capacities, and supply or demand amounts, but it is related to the network topology, the sets of source and destination nodes, and interdiction costs on each node and arc. We also observe that the critical budget $R_{a}$ that cannot make any demand be unsatisfied is related to all of these parameters of the network. Additionally, we present formulations to estimate both $R_{a}$ and $R_{b}$. For the effects of budget increasing, we presents conditions under which there would be extra capabilities to interdict more arcs and nodes with increased budget, and also under which the increased budget has no effects for the interdictor.

The remainder of this paper is organized as follows. In Section 2, models for network interdiction of multicommodity flows are presented. Additionally, we present the approaches to exactly solve the bilevel programs for both continuous and binary interdiction models. In Section 3, we perform the vulnerability analysis for the interdiction and analyze the unsatisfied demand amounts under different interdiction budgets, especially we study the critical budgets and effects of budget increasing. In Section 4, numerical experiments are performed to compare the proposed models and algorithms, and to analyze the results of vulnerability regarding different budgets. Finally, we conclude this paper in Section 5 with some future research directions.

\section{Models and Algorithms for Network Interdiction Problem}

The network interdiction problem has been extensively studied in [24]. Here we only give a brief introduction of the problem with both node and arc interdictions. Then we will focus on our new approach to solve the network interdiction problem for continuous interdiction case. These models and algorithms will provide fundamental methods for our analysis of interdiction in Section 3.

Let $G=(N, A)$ be a directed network consisting of node set $N$ (indexed by $i$ ) and arc set $A$ (each arc with head $j \in N$ and tail $i \in N$ is denoted by $(i, j)$ ). For any arc $(i, j) \in A$, assume the capacity is $u_{i j}$ and the cost to transport one unit of flow on this arc is $c_{i j}$. Additionally, assume the capacity for each node $i \in N$ is $v_{i}$. In this paper, we consider $K$ types of commodity flows (also, $K$ is used as the set of commodity types). For each type $k \in K$, let $S(k)$ denote the set of source nodes, and $D(k)$ the set of destination nodes. The supply amount of commodity $k$ at source node $i \in S(k)$ is $s_{i}^{k}$ while the demand amount at destination node $j \in D(k)$ is $d_{j}^{k}$. In the following, we assume that $s_{i}^{k}, d_{j}^{k}>0$ for all $i \in S(k), j \in D(k), k \in K$ and $\sum_{i \in S(k)} s_{i}^{k}=\sum_{j \in D(k)} d_{j}^{k}$ for all $k \in K$.

Let continuous decision variable $z_{i j}^{k}$ denote the commodity flow of type $k \in K$ on the $\operatorname{arc}(i, j) \in A$, and let $\mathbf{z}$ be the vector consisting of $z_{i j}^{k}$ 's for all $(i, j) \in A, k \in K$. The minimum-cost multicommodity network flow (MCNF) problem is to find possible flows to satisfy all demands with minimum cost for transhipment, and it can be 
formulated as a linear program in the following:

$$
\begin{array}{ll}
\min _{\mathbf{z}} & \sum_{(i, j) \in A} c_{i j} \sum_{k \in K} z_{i j}^{k} \\
\text { s.t. } & \sum_{j:(i, j) \in A} z_{i j}^{k}-\sum_{j:(j, i) \in A} z_{j i}^{k}=\left\{\begin{array}{ll}
s_{i}^{k}, & i \in S(k), \\
-d_{i}^{k}, & i \in D(k), \\
0, & i \notin\{S(k), D(k)\},
\end{array} \quad \forall i \in N, k \in K\right. \\
& \sum_{j:(i, j) \in A} \sum_{k \in K} z_{i j}^{k} \leq v_{i}, \quad \forall i \in N \\
& \sum_{k \in K} z_{i j}^{k} \leq u_{i j}, \quad \forall(i, j) \in A \\
& z_{i j}^{k} \geq 0, \forall(i, j) \in A, k \in K
\end{array}
$$

where the objective function (1a) is to minimize the cost associated to transport all commodities to satisfy the demand. Constraints (1b) ensure that the required amount of demand is shipped from each source to each destination and the flow balance is enforced for transhipment nodes. Constraints (1c) enforce that the total amount of flows that come out of each node cannot exceed the node capacity. This holds true for the flows that come into each node implicitly, except for destination node. For destination node $i$, let $v_{i}$ be the node capacity minus the total demand of this node, and therefore constraints (1c) also limit the total flow through vertex $i$. Constraints (1d) limit the total amount of flow on each arc by its capacity. Finally, constraints (1e) guarantee the nonnegativity requirements on the flows.

The network interdiction problem includes two roles, an interdictor and an operator. The interdictor, with a budget $R$, can attack the network (by removing nodes/arcs, or reducing their capacities) to disrupt the operation of the network, measured in terms of maximizing total flow cost. For any way the interdictor destroys the network without exceeding the budget, there exists an optimal commodity flow to satisfy all or a fraction of the demands in the resulted network decided by the operator. In the network interdiction models, the interdictor will choose the most severe way to destroy the network, in which the optimal cost is greater than the cost of any other way. This problem is usually formulated as a bilevel program.

\subsection{Models of network interdiction problem}

Let $x_{i}\left(0 \leq x_{i} \leq 1\right)$ denote the fraction of removal of node capacity $v_{i}$ by interdiction, and let $y_{i j}\left(0 \leq y_{i j} \leq 1\right)$ denote the fraction of removal of arc capacity $u_{i j}$. For convenience, let $\mathbf{x}, \mathbf{y}$ be the vectors for interdictions on nodes and arcs, respectively.

The interdiction models of multicommodity network flow can be formulated as a bilelvel program in the following:

$$
\max _{(\mathbf{x}, \mathbf{y}) \in \mathscr{I}} \min _{\mathbf{z}} \sum_{(i, j) \in A} c_{i j} \sum_{k \in K} z_{i j}^{k}
$$




$$
\begin{array}{ll}
\text { s.t. } & \sum_{j:(i, j) \in A} z_{i j}^{k}-\sum_{j:(j, i) \in A} z_{j i}^{k}=\left\{\begin{array}{ll}
s_{i}^{k}, & i \in S(k), \\
-d_{i}^{k}, & i \in D(k), \\
0, & i \notin\{S(k), D(k)\},
\end{array} \quad \forall i \in N, k \in K\right. \\
& \sum_{j:(i, j) \in A} \sum_{k \in K} z_{i j}^{k} \leq v_{i}\left(1-x_{i}\right), \quad \forall i \in N \\
& \sum_{k \in K} z_{i j}^{k} \leq u_{i j}\left(1-y_{i j}\right), \quad \forall(i, j) \in A \\
& z_{i j}^{k} \geq 0, \forall(i, j) \in A, k \in K
\end{array}
$$

where the objective function (2a) is to minimize the transport cost in resulted network under the worst-case interdiction. The decision variables $\mathbf{x}, \mathbf{y}$ in the outer level of maximization are to find the worst-case interdiction with limited budget $R$. The constraints for the interdictor are denoted in the set $\mathscr{I}$. Constraints $(2 \mathrm{~b})$ ensure that the required amount of demand is shipped from each source to each destination and the flow balance is enforced for transhipment nodes. The flow amounts are limited in the resulted network after some disruption on some arcs or nodes or decrease of capacities, given by constraints (2c)-(2d). Constraints (2c) present the node capacity limits after decreasing the capacity by interdiction, and constraints $(2 \mathrm{~d})$ present the remaining capacity limit of each arc after the interdiction. The last constraints (2e) are the nonnegativity requirements for all flows.

If the set $\mathscr{I}$ for interdiction limitation is chosen as

$$
\begin{aligned}
\mathscr{I}_{c}=\left\{(\mathbf{x}, \mathbf{y}) \in \mathbb{R}^{|N|+|A|}:\right. & \sum_{i \in N} p_{i} x_{i}+\sum_{(i, j) \in A} q_{i j} y_{i j} \leq R, \\
& \left.0 \leq x_{i}, y_{i j} \leq 1, \forall i \in N,(i, j) \in A\right\},
\end{aligned}
$$

where $R$ is total budget or resource for interdiction, and $p_{i}$ and $q_{i j}$ are the amount of budget (or called interdiction cost) used for destroying node $i \in N$ and $\operatorname{arc}(i, j) \in$ $A$, respectively, and the formulation (2) becomes a continuous interdiction model. Mainly, the node and arc capacities are lowered by interdiction.

If $\mathscr{I}$ is chosen as

$$
\mathscr{I}_{b}=\left\{(\mathbf{x}, \mathbf{y}) \in\{0,1\}^{|N|+|A|}: \sum_{i \in N} p_{i} x_{i}+\sum_{(i, j) \in A} q_{i j} y_{i j} \leq R\right\},
$$

the formulation (2) is a binary interdiction model. Instead of partially destroying some nodes or arcs, the interdictor completely destroys the nodes and/or arcs. Note that the binary interdiction model can be considered as a special case of continuous interdiction model.

In some models, there exist arc-only interdictions (e.g., [24]), and the corresponding constraint in $\mathscr{I}_{c}$ and $\mathscr{I}_{b}$ is $\sum_{(i, j) \in A} q_{i j} y_{i j} \leq R$. In the following, we consider the general situation. Following the node splitting technique introduced in [32] (pp. 4142), the transformed network for $G=(N, A)$ is defined by $G^{\prime}=\left(N^{\prime}, A^{\prime}\right)$ with node set $N^{\prime}=\left\{i_{1}, i_{2}: \forall i \in N\right\}$ and $\operatorname{arc} \operatorname{set} A^{\prime}=\left\{\left(i_{1}, i_{2}\right): \forall i \in N\right\} \cup\left\{\left(i_{2}, j_{1}\right), \forall(i, j) \in A\right\}$ with arc transport cost $c_{i_{1}, i_{2}}^{\prime}=0$ and arc capacity $u_{i_{1}, i_{2}}^{\prime}=v_{i}$ for any $i \in A$, and arc transport cost $c_{i_{2}, j_{1}}^{\prime}=c_{i j}$ and arc capacity $u_{i_{2}, j_{1}}^{\prime}=u_{i j}$ for any $(i, j) \in A$. There is no 
node capacity or node interdiction in the network $G^{\prime}$. Any source node $i \in S(k)$ for all $k \in K$ in $G$ now becomes $i_{1}$ in $G^{\prime}$, with supply amount $s_{i_{1}}^{\prime k}=s_{i}^{k}$ while node $i_{2}$ is just a transhipment node. Any destination node $i \in D(k)$ for all $k \in K$ in $G$ now becomes $i_{1}$ in $G^{\prime}$, with demand amount ${d^{\prime}}_{i_{1}}^{k}=d_{i}^{k}$ while node $i_{2}$ is just a transhipment node. The interdiction cost $q_{i_{1}, i_{2}}^{\prime}$ in $G^{\prime}$ is assumed to be $p_{i}$ of node $i$ in $G$, and the interdiction cost $q_{i_{2}, j_{1}}^{\prime}$ in $G^{\prime}$ is assumed to be $q_{i j}$ of $\operatorname{arc}(i, j)$ in $G$.

The network $G^{\prime}$ can give optimal flows with only arc capacities to satisfy the demand. Also, interdiction on network $G^{\prime}$ is equivalent to the interdiction on network $G$. We have the following property (its proof is presented in Appendix A).

Proposition 1 For both continuous and binary interdictions, the models (2) on network $G=(N, A)$ with interdiction on both nodes and arcs are equivalent to corresponding models on network $G^{\prime}=\left(N^{\prime}, A^{\prime}\right)$ with interdiction only on arcs, with the same budget.

Intuitively, if the available budget $R$ is large enough, all demands in the network cannot be satisfied. On the other hand, if $R$ is relatively small, all demands can be satisfied in the resulted network after some node/arc removals, but the cost for transport is increased. In the following Sections 2.2, we assume that $R$ is relatively small and all demands can still be satisfied after interdiction. The solution approaches are proposed to solve formulation (2) in both continuous and binary interdiction models. In Section 3, we quantitatively analyze the effect of increasing budget, under which the demand can be partially satisfied or all demands cannot be satisfied.

\subsection{Approaches for binary and continuous interdiction}

In the binary case, the interdictor can only choose to completely destroy an arc/node or not, instead of partially destroying the capability of the arc/node. Thus $\mathbf{x}, \mathbf{y}$ are binary, and are limited by $\mathscr{I}_{b}$. For fixed $\mathbf{x}, \mathbf{y}$ chosen in $\mathscr{I}_{b}$, which denote an interdiction plan, we consider the dual problem for inner problem. Let $\alpha_{i k}$, for any $i \in N, k \in K$, be the dual variables associated with constraints (2b) and $\beta_{i}, \gamma_{i j}$, for any $i \in N,(i, j) \in A$, be the dual variables associated with constraints (2c) and (2d), respectively. Let $\alpha, \beta$, $\gamma$ be corresponding vectors formed by all $\alpha_{i k}$ 's, $\beta_{i}$ 's, $\gamma_{i j}$ 's. If for any $(\mathbf{x}, \mathbf{y}) \in \mathscr{I}_{c}$, the inner problem is feasible, we can use strong duality and combine it with outer level to obtain the following equivalent model for binary interdiction:

$$
\begin{aligned}
\max _{\mathbf{x}, \mathbf{y} ; \alpha, \beta, \gamma} & \sum_{k \in K} \sum_{i \in S(k)} s_{i}^{k} \alpha_{i k}-\sum_{k \in K} \sum_{i \in D(k)} d_{i}^{k} \alpha_{i k}+\sum_{i \in N} v_{i}\left(1-x_{i}\right) \beta_{i}+\sum_{(i, j) \in A} u_{i j}\left(1-y_{i j}\right) \gamma_{i j} \\
\text { s.t. } & \alpha_{i k}-\alpha_{j k}+\beta_{i}+\gamma_{i j} \leq c_{i j}, \forall k \in K,(i, j) \in A \\
& \beta_{i}, \gamma_{i j} \leq 0, \forall i \in N,(i, j) \in A \\
& \sum_{i \in N} p_{i} x_{i}+\sum_{(i, j) \in A} q_{i j} y_{i j} \leq R \\
& x_{i}, y_{i j} \in\{0,1\}, \forall i \in N,(i, j) \in A
\end{aligned}
$$


The approach is used in [24]. The nonlinear terms $\left(1-x_{i}\right) \beta_{i},\left(1-y_{i j}\right) \gamma_{i j}$ are products of one binary variable and one continuous variable. By introducing $\eta_{i}=$ $\left(1-x_{i}\right) \beta_{i}, \zeta_{i j}=\left(1-y_{i j}\right) \gamma_{i j}$ to replace them in the objective and adding following constraints, we can linearize the problem:

$$
\begin{aligned}
& \eta_{i} \leq 0, \eta_{i} \leq \beta_{i}+M x_{i}, \eta_{i} \geq-M\left(1-x_{i}\right), \eta_{i} \geq \beta_{i}, \forall i \in N \\
& \zeta_{i j} \leq 0, \zeta_{i j} \leq \gamma_{i j}+M y_{i j}, \zeta_{i j} \geq-M\left(1-y_{i j}\right), \zeta_{i j} \geq \gamma_{i j}, \forall(i, j) \in A
\end{aligned}
$$

in which the " $\geq$ " type of constraints can be eliminated as this is a maximization problem and they are redundant.

Consider the continuous interdiction model, i.e., $\mathscr{I}$ is chosen as $\mathscr{I}_{c}$ in formulation (2), we propose an algorithm to solve this problem, and presented in the following theorem (its proof is presented in Appendix B).

Theorem 1 Assume that the budget $R$ satisfies that all demands can be satisfied for any continuous interdiction plans in $\mathscr{I}_{c}$. formulation (2) can be formulated equivalently in the following mixed integer program:

$$
\begin{aligned}
\max _{\delta, \delta^{\prime}, \sigma, \sigma^{\prime}, \alpha, \beta, \gamma} & \sum_{k \in K}\left(\sum_{i \in O(k)} s_{i}^{k} \alpha_{i k}-\sum_{i \in D(k)} d_{i}^{k} \alpha_{i k}\right) \\
& +\sum_{i \in N} \beta_{i}\left(1-\delta_{i}-\delta_{i}^{\prime} R^{\prime} / p_{i}\right)+\sum_{(i, j) \in A} \gamma_{i j}\left(1-\sigma_{i j}-\sigma_{i j}^{\prime} R^{\prime} / q_{i j}\right) \\
\text { s.t. } & \alpha_{i k}-\alpha_{j k}+\beta_{i} / v_{i}+\gamma_{i j} / u_{i j} \leq c_{i j}, \forall(i, j) \in A, \forall k \in K \\
& \left\{\begin{array}{l}
R^{\prime} / p_{i} \leq 1+M_{i}\left(1-\delta_{i}^{\prime}\right), \forall i \in N \\
R^{\prime} / q_{i j} \leq 1+M_{i j}\left(1-\sigma_{i j}^{\prime}\right), \forall(i, j) \in A
\end{array}\right. \\
& \sum_{i \in N} p_{i} \delta_{i}+\sum_{(i, j) \in A} q_{i j} \sigma_{i j}+R^{\prime}=R \\
& \delta_{i}+\delta_{i}^{\prime} \leq 1, \forall i \in N \\
& \sigma_{i j}+\sigma_{i j}^{\prime} \leq 1, \forall(i, j) \in A \\
& \sum_{i \in N} \delta_{i}^{\prime}+\sum_{(i, j) \in A} \sigma_{i j}^{\prime}=1 \\
& \delta_{i}, \sigma_{i j}, \delta_{i}^{\prime}, \sigma_{i j}^{\prime} \in\{0,1\}, \forall i \in N,(i, j) \in A \\
& R^{\prime} \geq 0 ; \beta_{i}, \gamma_{i j} \leq 0, \forall(i, j) \in A, \forall k \in K
\end{aligned}
$$

The terms $\delta_{i} \beta_{i}, \sigma_{i j} \gamma_{i j}, \beta_{i} \delta_{i}^{\prime} R^{\prime}, \gamma_{i j} \sigma_{i j}^{\prime} R^{\prime}$ in the objective (B.5a) in formulation (B.5) are nonlinear. The first two terms can be linearized by approaches introduced in (6). As $R^{\prime}=R-\left(\sum_{i \in N} p_{i} \delta_{i}+\sum_{(i, j) \in A} q_{i j} \sigma_{i j}\right)$ in the other two terms is actually expressed by binary variables, and thus $\beta_{i} \delta_{i}^{\prime} R^{\prime}, \gamma_{i j} \sigma_{i j}^{\prime} R^{\prime}$ actually include nonlinear terms as product of two binary variables and one continuous variable. This type of product can be further linearized by introducing one more binary variable as product of these two binary ones. For example, one $\delta_{i}^{\prime} \delta_{j} \beta_{i}$ in $\beta_{i} \delta_{i}^{\prime} R^{\prime}$ can be converted to $\tau_{i j} \beta_{i}$ (which can be linearized by approaches (6)), in which $\tau_{i j}=\delta_{i}^{\prime} \delta_{j}$ can be linearized by adding constraints $\tau_{i j} \leq \delta_{i}, \tau_{i j} \leq \delta_{j}^{\prime}$ as the objective is to maximize. 


\section{Interdiction Budget Analysis}

Intuitively, if the budget $R$ is large enough, all demands in the network cannot be satisfied. On the other direction, if $R$ is relatively small, all demands can still be satisfied in the resulted network after some node/arc removals, but the cost for transport is increased. Therefore, there exists two critical values $R_{a}, R_{b}\left(R_{a}<R_{b}\right)$, such that if $0 \leq R \leq R_{a}$, all demands should be satisfied, and if $R \geq R_{b}$, none of the demands can be satisfied. For a budget $R_{a}<R<R_{b}$, only a fraction of the demands can be satisfied.

\subsection{Unsatisfied demands regarding budget $R$}

Assume that $\lambda_{i}^{k}$ denotes the amount of unsatisfied demand at node $i \in D(k)$ of type $k$ commodity. Also, let $\lambda_{i}^{\prime k}$ denote the unused supply at node $i \in S(k)$ of type $k$ commodity. Therefore, $\sum_{k \in K} \sum_{i \in D(k)} \lambda_{i}^{k}$ denotes the total unsatisfied demand in the resulted network after interdiction. In the following, when we analyze the critical budgets, we assume that under any interdiction plan, the operator always tries to minimize the unsatisfied demand. Thus, the following model $\mathbf{U D}(R)$ can be used to find the minimum unsatisfied demand under the worst-case interdiction with given budget $R$ :

$$
\begin{aligned}
& \mathbf{U D}(R) \text { : } \\
& F(R)=\max _{(\mathbf{x}, \mathbf{y}) \in \mathscr{I}} \min _{\mathbf{z}, \lambda} \sum_{k \in K} \sum_{i \in D(k)} \lambda_{i}^{k} \\
& \text { s.t. } \sum_{j:(i, j) \in A} z_{i j}^{k}-\sum_{j:(j, i) \in A} z_{j i}^{k}=\left\{\begin{array}{ll}
s_{i}^{k}-\lambda_{i}^{\prime k}, & i \in S(k), \\
-d_{i}^{k}+\lambda_{i}^{k}, & i \in D(k), \\
0, & i \notin\{S(k), D(k)\},
\end{array} \quad \forall i \in N, k \in K\right. \\
& \sum_{j:(i, j) \in A} \sum_{k} z_{i j}^{k} \leq v_{i}\left(1-x_{i}\right), \quad \forall i \in N \\
& \sum_{k \in K} z_{i j}^{k} \leq u_{i j}\left(1-y_{i j}\right), \quad \forall(i, j) \in A \\
& 0 \leq \lambda_{i}^{\prime k} \leq s_{i}^{k}, \forall k \in K, \forall i \in S(k) \\
& 0 \leq \lambda_{i}^{k} \leq d_{i}^{k}, \forall k \in K, \forall i \in D(k) \\
& z_{i j}^{k} \geq 0, \forall(i, j) \in A, k \in K
\end{aligned}
$$

where the optimal objective value $F(R)$ is a function of the budget $R$ for the amount of unsatisfied demand. In formulation (8), the decision variables $\mathbf{x}, \mathbf{y}$ for interdiction and $\mathbf{z}$ for flows in the resulted network are the same as those in formulation (2). The objective is to obtain the minimum unsatisfied demand under the worse-case interdiction. In constraints $(8 \mathrm{~b}), s_{i}^{k}-\lambda_{i}^{\prime k}$ is the amount of commodities that are successfully transported from source node $i$, and $d_{i}^{k}-\lambda_{i}^{k}$ is the amount of satisfied demand at destination node $i$. For transhipment node, the balance flow constraints are enforced. Constraints $(8 \mathrm{e})$ is to ensure the amount of commodities that are not transported from 
source node $i$ cannot exceed its supply. Similarly, constraints (8f) guarantee the unsatisfied demand at destination node $i$ cannot exceed its demand. The rest constraints have the same meaning as those in formulation (2).

In formulation (8), for any interdiction $(\mathbf{x}, \mathbf{y}) \in \mathscr{I}$, the inner problem to find minimum unsatisfied demand is always feasible. To solve this problem, for binary case, we can obtain the dual of inner problem and combine it with outer level as the method we introduced in Section 2.2. Then we can introduce new variables to linearize the nonlinear terms consisting of products of one binary variable and one continuous variable. After that, formulation (8) becomes a mixed integer linear program. For continuous case, the conclusion in Section 2.2 that the condition "except one node or arc being partially interdicted, the rest interdictions are binary" still holds, and we can use the same approach introduced in Section 2.2 to solve (8).

Now, for a given network $G=(N, A)$, with node capacity $v_{i}$ for all $i \in N$ and arc capacity for all arc $(i, j) \in A$, sources $S(k)$ and destinations $D(k)$ for all commodity $k \in K$, supply amount $s_{i}^{k}$ for all $i \in S(k), k \in K$ and demand amount $d_{j}^{k}$ for all $j \in$ $D(k), k \in K$, and interdiction cost $p_{i}$ for all $i \in A$ and $q_{i j}$ for all $(i, j) \in A$, we define two critical budgets for interdiction. The critical budget $R_{a}$ is the largest value under which all demands can still be satisfied in the resulted network even under the worstcase interdiction, and the critical budget $R_{b}$ is the least value under which the worstcase interdiction can make none of the demands be satisfied. Therefore, for $0 \leq R \leq$ $R_{a}, F(R)=0$; for $R_{a}<R<R_{b}, 0<F(R)<\sum_{k \in K} \sum_{i \in D(k)} d_{i}^{k}$; for $R \geq R_{b}, F(R)=$ $\sum_{k \in K} \sum_{i \in D(k)} d_{i}^{k}$.

As an example, we test both binary and continuous interdiction models on a network with 8 nodes, 30 arcs, 3 commodities and show the result of binary interdiction for $F(R)$ regarding different interdiction budgets in Fig. 1(a) and result of continuous interdiction in Fig. 1(b). For binary interdiction, when $R \leq 2, F(R)=0$. Thus for any interdiction under budget $R$, all demands are satisfied. When $R>2$, there exist unsatisfied demands. Thus $R=2$ is the largest value where all demands can be satisfied and critical budget $R_{a}=2$. When $2<R<8,0<F(R)<\sum_{k \in K} \sum_{i \in D(k)} d_{i}^{k}$, only part of the demands can be satisfied. For $R \geq 8, F(R)=\sum_{k \in K} \sum_{i \in D(k)} d_{i}^{k}$, no demand can be satisfied. $R=8$ is the least value of making all demands unsatisfied, and thus critical budget $R_{b}=8$. For continuous interdiction, we find the critical budgets $R_{a}=1.2$ and $R_{b}=8$. We can observe that the critical budget $R_{a}$ for continuous interdiction is larger that that of binary interdiction, but their critical budgets $R_{b}$ are the same.

Proposition 2 For both continuous and binary interdictions regarding different budgets $R \geq 0$ in formulation (8), the function $F(R)$ has the following properties:

(i) For a budget $R \geq 0$, if $F(R)=0$, the inner level of corresponding interdiction (2) is feasible for any interdiction plan $\mathbf{x}, \mathbf{y}$; otherwise, $F(R)>0$, the inner level of (2) is infeasible for some interdiction plan $\mathbf{x}, \mathbf{y}$.

(ii) For the same network and any budget $R \geq 0$, the value $F(R)$ under continuous interdiction is larger than or equal to the corresponding value under binary interdiction.

(iii) The function $F(R)$ is monotonically increasing on $R \in[0,+\infty)$.

(iv) The function $F(R)$ for continuous interdiction is a continuous function, while $F(R)$ for binary interdiction is upper semicontinuous on $R \in[0,+\infty)$. 


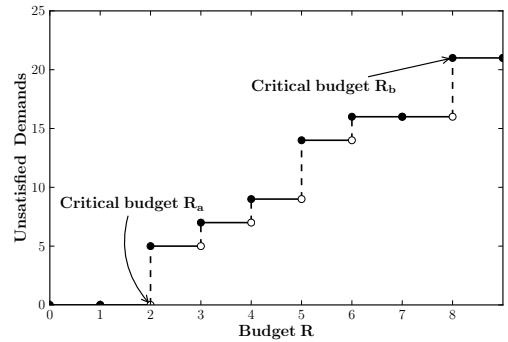

(a) binary interdiction

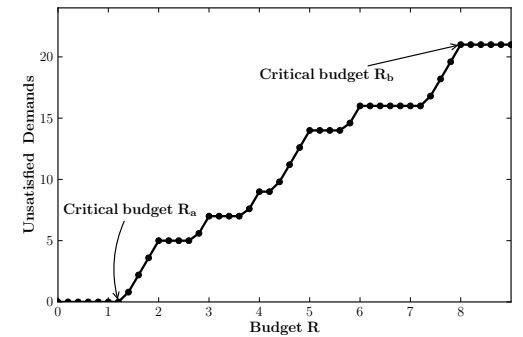

(b) continuous interdiction

Fig. 1 Unsatisfied demands and interdiction budgets

For (i), if $F(R)=0$ for a budget $R \geq 0$, no matter how the interdictor destroys the network, all demands will be satisfied. That is for this budget $R$, for any $\mathbf{x}, \mathbf{y} \in \mathscr{I}$, inner level of (2) is always feasible; otherwise, $F(R)>0$, which means there exists an $\mathbf{x}, \mathbf{y}$ such that some demands will be not satisfied. That is, for some $\mathbf{x}, \mathbf{y} \in \mathscr{I}$, the inner level of (2) will be infeasible. Therefore, the approaches by duality introduced in Section 2.2 to solve formulation (2) can only be applied for cases with $R \leq R_{a}$, as when $R>R_{a}$, the inner problem of (2) is infeasible for worst-case interdiction $\mathbf{x}, \mathbf{y}$ under budget $R$.

For (ii), let $F^{(b)}(R), F^{(c)}(R)$ be the value of $F(R)$ for network $G$ to be interdicted under binary and continuous interdictions with the same budget $R$, respectively. With budget $R$, any binary interdiction $\left(\mathbf{x}^{(b)}, \mathbf{y}^{(b)}\right) \in \mathscr{I}_{b}$ is also feasible for continuous interdiction with same budget, i.e., $\left(\mathbf{x}^{(b)}, \mathbf{y}^{(b)}\right) \in \mathscr{I}_{c}$. Thus, the worst-case binary interdiction is not necessary to be a worst-case continuous interdiction. In the resulted network under the worst-case interdiction, the satisfied amount under binary interdiction should be less than or equal to the amount under continuous interdiction, i,e., $F^{(b)}(R) \leq F^{(c)}(R)$.

For (iii), the function $F(R)$ is monotonically increasing. Because for budget constraint in $\mathscr{I}$, if budget $R$ increases, all former interdiction plans under $R$ are also feasible under $R+\Delta R$, with some possible additional disruptions on nodes and/or arcs. Thus, the unsatisfied demands should be nondecreasing, i.e., $F(R) \leq F(R+\Delta R)$ for any $\Delta R \geq 0$.

For (iv), in the continuous interdiction case, for any $R_{0}>0$ and some $\varepsilon \geq 0$, we have $F\left(R_{0}\right)=\lim _{\mathcal{E} \rightarrow 0} F\left(R_{0}+\varepsilon\right)$ and also $F\left(R_{0}\right)=\lim _{\mathcal{E} \rightarrow 0} F\left(R_{0}-\varepsilon\right)$, which imply that $F(R)$ is a continuous function on $R \geq 0$ with consideration of right-continuous at $R=0$. Proposition 2(iv) is also discussed in Section 3.3. For an example of Proposition 2(ii)-(iv), we refer to Fig. 1.

For the interdictor, with a budget $R \leq R_{a}$, whatever the interdiction plan is, there always exists a feasible flow in the resulted network to satisfy all demands; if the budget $R \geq R_{b}$, there always exists an interdiction plan (the worst-case one) that makes all demands be unsatisfied. Thus, by increasing of budget to a value that is still under budget $R_{a}$ or larger than $R_{b}$, there are no extra benefits for the interdictor. However, for a budget $R \in\left(R_{a}, R_{b}\right)$, increase of budget may give the interdictor extra capability 
to destroy the network, i.e., increasing the amount of unsatisfied demands. In the following, we first present approaches to compute the critical budgets $R_{a}, R_{b}$ and then study the effects when budget increases.

\subsection{Computing critical budgets}

From Proposition 2(iii), the curve for $F(R)$ is monotonically increasing on $R \in[0,+\infty)$, where the point $R_{a}$ is largest point with $F(R)=F\left(R_{a}\right)=0$ and $R_{b}$ is smallest point with $F(R)=F\left(R_{b}\right)=\sum_{k \in K} \sum_{i \in D(k)} d_{i}^{k}$. As the inner problem of (2) will become infeasible for the worst-case interdiction $\mathbf{x}, \mathbf{y}$ when $R>R_{a}$, and the dual problem will be unbounded. Thus, techniques introduced in Section 2.2 for solving (2) can be used for obtaining $R_{a}$ by gradually increasing budget $R$ from 0 till some value where total cost increases to an abnormally large number, which should be larger than its bound (For example, one option for this bound is $\left.\sum_{i \in N} \max _{(i, j) \in A} c_{i j} \sum_{k \in K} \sum_{i \in D(k)} d_{i}^{k}\right)$. This value of $R$ is $R_{a}$. On the other hand, formulation (8) can be used to obtain $R_{a}, R_{b}$. However, this requires heavy computations on testing different values of $R$. In the following, we present properties for $R_{a}, R_{b}$ in different networks and some direct methods to compute them.

\subsubsection{Critical budget $R_{b}$}

Theorem 2 In network $G=(N, A)$, with node capacity $v_{i}$ for all $i \in N$ and arc capacity for all arc $(i, j) \in A$, and sources $S(k)$ and destinations $D(k)$ for all commodity $k \in K$, the critical budget values $R_{b}$ under the the fixed interdiction costs $p_{i}, q_{i j}$ for all $i \in N,(i, j) \in A$, for both continuous interdiction and binary interdiction models are the same.

Proof. Let $R_{b}^{(b)}, R_{b}^{(c)}$ be the critical budget $R_{b}$ for network $G$ to be interdicted under binary and continuous interdictions, respectively. Since $R_{b}^{(b)}$ is the critical budget, there must be a binary interdiction $\left(\mathbf{x}^{(b)}, \mathbf{y}^{(b)}\right) \in \mathscr{I}_{b}$ that makes all demand in the resulted network be unsatisfied. As this plan is also feasible for continuous interdiction, i.e., $\left(\mathbf{x}^{(b)}, \mathbf{y}^{(b)}\right) \in \mathscr{I}_{c}$ with $R=R_{b}^{(b)}$, the inequality $R_{b}^{(c)} \leq R_{b}^{(b)}$ holds.

On the other hand, as $R_{c}^{(c)}$ is the critical budget, for any interdiction $\left(\mathbf{x}^{(c)}, \mathbf{y}^{(c)}\right) \in$ $\mathscr{I}_{c}$ that makes all demand in the resulted network be unsatisfied, we claim that $\left(\mathbf{x}^{(c)}, \mathbf{y}^{(c)}\right) \in \mathscr{I}_{b}$ with $R=R_{b}^{(c)}$, i.e., we need to show that elements of $\mathbf{x}^{(c)}, \mathbf{y}^{(c)}$ are all binary. From Theorem 1, here we only need to show the case for arc interdiction $\mathbf{y}^{(c)}$. By contradiction, if there exists one arc $\left(i^{\prime}, j^{\prime}\right)$ with fractional interdiction, i.e., $0<y_{i^{\prime} j^{\prime}}^{(c)}<1$, for some $\varepsilon \in\left(0, y_{i^{\prime} j^{\prime}}^{(c)}\right)$, there exists another interdiction with less budget but same interdiction approaches on all nodes and arcs except on $\operatorname{arc}\left(i^{\prime}, j^{\prime}\right)$, which is interdicted to have remaining capacity $u_{i^{\prime} j^{\prime}}\left(1-\left(y_{i^{\prime} j^{\prime}}^{(c)}-\varepsilon\right)\right)$. This new interdiction plan has less budget than $R_{b}^{(c)}$, thus some demand can be satisfied by flow through arc $\left(i^{\prime}, j^{\prime}\right)$, bounded by the capacity $u_{i^{\prime} j^{\prime}}\left(1-\left(y_{i^{\prime} j^{\prime}}^{(c)}-\varepsilon\right)\right)$, which means that there exists some type of commodity that its demand can be partially satisfied. 
We claim that there exists one $k$, such that for some $i \in S(k), j \in D(k)$, there is a flow $f$ from $i$ to $i^{\prime}$, then through $\left(i^{\prime}, j^{\prime}\right)$ and then $j^{\prime}$ to $j$. In fact, the resulted networks, either by interdiction $\left(\mathbf{x}^{(c)}, \mathbf{y}^{(c)}\right)$ or same interdiction except the one on arc $\left(i^{\prime}, j^{\prime}\right)$ which is interdicted by $y_{i^{\prime} j^{\prime}}^{(c)}-\varepsilon$, are the same except arc arc $\left(i^{\prime}, j^{\prime}\right)$. If the flow $f$ does not pass through arc $\left(i^{\prime}, j^{\prime}\right)$, there exists the same flow from $i$ to $j$ in both resulted networks. This is a contradiction to the fact the interdiction $\left(\mathbf{x}^{(c)}, \mathbf{y}^{(c)}\right)$ make all demands be unsatisfied.

Thus in the resulted network with interdiction $y_{i^{\prime} j^{\prime}}^{(c)}$, there still exists a flow with amount $\min \left\{f, u_{i^{\prime} j^{\prime}}\left(1-y_{i^{\prime} j^{\prime}}^{(c)}\right)\right\}$ through $\left(i^{\prime}, j^{\prime}\right)$, to satisfy some demand at node $j$ from node $i$, which is a contradiction to that no demand can be satisfied under interdiction $\left(\mathbf{x}^{(c)}, \mathbf{y}^{(c)}\right) \in \mathscr{I}_{c}$ with budget $R_{c}^{(c)}$. Therefore, all elements of $\mathbf{x}^{(c)}, \mathbf{y}^{(c)}$ are all binary, that is $\left(\mathbf{x}^{(c)}, \mathbf{y}^{(c)}\right) \in \mathscr{I}_{b}$ with $R=R_{b}^{(c)}$. Now the inequality $R_{b}^{(c)} \geq R_{b}^{(b)}$ holds.

Therefore, the critical budgets $R_{b}$ are the same for both continuous and binary interdictions on the same network.

In the following, by Theorem 2, we do not classify the cases of continuous and binary interdictions during studying properties of critical budget $R_{b}$.

Lemma 1 In network $G=(N, A)$, with node capacity $v_{i}$ for all $i \in N$ and arc capacity $u_{i j}$ for all arc $(i, j) \in A$, and sources $S(k)$ and destinations $D(k)$ for all commodity $k \in K$, the critical budget value $R_{b}$ under interdiction costs $p_{i}, q_{i j}$ for all $i \in N,(i, j) \in$ $A$, is the same for any supply amount $s_{i}^{k}$ and demand amount $d_{j}^{k}$, for all $i \in S(k), j \in$ $D(k), k \in K$, that this network can transport.

Proof. Without loss of generality, we assume two networks $G_{1}$ and $G_{2}$ are constructed based on $G=(N, A)$ with same topology structure, capacities and $S(k), D(k)$ for all $k \in K$ sets, but $G_{1}$ has supply/demand amounts $s_{i}^{(1) k}, d_{j}^{(1) k}$ and $G_{2}$ has $s_{i}^{(2) k}, d_{j}^{(2) k}$ for all $i \in S(k), j \in D(k), k \in K$, where all demands can be met in both networks. We claim that $G_{1}$ and $G_{2}$ have the same critical budget $R_{b}$ if values of $p_{i}, q_{i j}$ for all $i \in N,(i, j) \in A$ in $G_{1}$ are the same as those corresponding ones in $G_{2}$.

Let $R_{b}^{(1)}$ be the critical budget $R_{b}$ for network $G_{1}$ and $R_{b}^{(2)}$ for network $G_{2}$. We claim that $R_{b}^{(1)}=R_{b}^{(2)}$. Otherwise, we have cases

- $R_{b}^{(1)}>R_{b}^{(2)}$. The budget $R_{b}^{(2)}$ is not large enough to destroy network $G_{1}$ to make all demands be unsatisfied. However, $R_{b}^{(2)}$ is the critical budget $R_{b}$ for $G_{2}$, then there must exist an interdiction $\mathbf{x}^{(2)}, \mathbf{y}^{(2)}$ which can destroy $G_{2}$ to make all $d_{j}^{(2) k}$ be unsatisfied. If the budget $R_{b}^{(2)}$ is used to destroy $G_{1}$ in the way of $\mathbf{x}^{(2)}, \mathbf{y}^{(2)}$, as $R_{b}^{(1)}>R_{b}^{(2)}$, the interdiction is not complete and there exists a flow from some source $i \in S(k)$ to destination $j \in D(k)$ for some $k$. The resulted networks destroyed by $R_{b}^{(2)}$ are the same, there exists a same flow in $G_{2}$, which is a contradiction to the fact that no demand can be satisfied in $G_{2}$ by interdiction $\mathbf{x}^{(2)}, \mathbf{y}^{(2)}$. Therefore, $R_{b}^{(1)}>R_{b}^{(2)}$ cannot hold.

$-R_{b}^{(1)}<R_{b}^{(2)}$. This can be proved similarly as above case.

Therefore, the two critical budgets of $R_{b}$ for $G_{1}$ and $G_{2}$ are the same. For all possible choices of $s_{i}^{k}, d_{j}^{k}$ 's, we finish the proof. 
Lemma 2 In network $G=(N, A)$, with node capacity $v_{i}$ for all $i \in N$ and arc capacity for all arc $(i, j) \in A$, and sources $S(k)$ and destinations $D(k)$ for all commodity $k \in K$, if interdiction costs $p_{i}$ for all $i \in N$ and $q_{i j}$ for all $(i, j) \in A$ are fixed, the changes of node and arc capacities $v_{i}, u_{i j}$ do not change the critical budget $R_{b}$.

Proof. Without loss of generality, we assume two networks $G_{1}$ and $G_{2}$ are constructed based on $G=(N, A)$ with same parameters, including interdiction cost $p_{i}$ on each node and $q_{i j}$ on each arc, but with different node and arc capacities.

First we show that $G_{1}$ and $G_{2}$ have the same supply and demand amounts, and have only one $\operatorname{arc}(i, j)$ with different capacities, $u_{i j}^{(1)}$ in $G_{1}$ and $u_{i j}^{(2)}$ in $G_{2}\left(u_{i j}^{(1)} \neq u_{i j}^{(2)}\right)$. Without loss of generality, we assume $0<u_{i j}^{(1)}<u_{i j}^{(2)}$, and that the critical budget $R_{b}$ in $G_{1}$ is $R_{b}^{(1)}$ and in $G_{2}$ is $R_{b}^{(2)}$. We claim that $R_{b}^{(1)}=R_{b}^{(2)}$.

In fact, if this does not hold, we have two cases:

$-R_{b}^{(1)}>R_{b}^{(2)}$. In this case, budget $R_{b}^{(2)}$ is not large enough to destroy network $G_{1}$ to make all demands be unsatisfied. However, because $R_{b}^{(2)}$ is critical budget $R_{b}$ for network $G_{2}$, we can find an interdiction way $\left(\mathbf{x}^{(2)}, \mathbf{y}^{(2)}\right)$, which can destroy $G_{2}$ to make all $d_{j}^{(2) k}$ be unsatisfied. Denote the resulted network after interdiction is $G_{2}^{\prime}$. If the budget $R_{b}^{(2)}$ is used to destroy $G_{1}$ in the way of $\left(\mathbf{x}^{(2)}, \mathbf{y}^{(2)}\right)$, as $R_{b}^{(1)}>R_{b}^{(2)}$, the interdiction is not complete and there exists a flow from some source $i \in S(k)$ to destination $j \in D(k)$ for some $k$. Denote this resulted network as $G_{1}^{\prime}$. The capacities of all nodes/arcs in $G_{1}^{\prime}$, except for $(i, j)$, is the same as the corresponding capacities in $G_{2}^{\prime}$. Because $u_{i j}^{(1)}<u_{i j}^{(2)}$, we have $u_{i j}^{(1)}\left(1-y_{i j}^{(2)}\right) \leq u_{i j}^{(2)}\left(1-y_{i j}^{(2)}\right)$. That is the remaining capacity of arc $(i, j)$ in $G_{1}$ is less than or equal to capacity of arc $(i, j)$ in $G_{2}$, and this is the only difference between $G_{1}^{\prime}$ and $G_{2}^{\prime}$. As there exists flow from $s$ to $t$ in $G_{1}^{\prime}$, there must exist a same flow from same source $i \in S(k)$ to destination $j \in D(k)$ in $G_{2}^{\prime}$, which is a contradiction to the fact that budget $R_{b}^{(2)}$ is critical budget $R_{b}$ for network $G_{2}$. Therefore $R_{b}^{(1)}>R_{b}^{(2)}$ cannot hold.

- $R_{b}^{(1)}<R_{b}^{(2)}$. In this case, budget $R_{b}^{(1)}$ is not large enough to destroy network $G_{2}$ to make all demands be unsatisfied. Because $R_{b}^{(1)}$ is critical budget $R_{b}$ for network $G_{1}$, we can find an interdiction way $\left(\mathbf{x}^{(1)}, \mathbf{y}^{(1)}\right)$, which can totally destroy network $G_{1}$ and denote the resulted network after interdiction is $G_{1}^{\prime}$. If the budget $R_{b}^{(1)}$ is used to destroy $G_{2}$ in the way of $\left(\mathbf{x}^{(1)}, \mathbf{y}^{(1)}\right)$, as $R_{b}^{(1)}<R_{b}^{(2)}$ in the resulted network $G_{2}^{\prime}$, the interdiction is not complete and there exists a flow $f_{2}$ from some source $i \in S(k)$ to destination $j \in D(k)$ for some $k$ in resulted network $G_{2}^{\prime}$. The capacities of all nodes/arcs in $G_{2}^{\prime}$, except for $(i, j)$, are the same as the corresponding ones in $G_{1}^{\prime}$. Because $u_{i j}^{(2)}>u_{i j}^{(1)}$, the remaining capacity of arc $(i, j)$ in $G_{2}$ is larger than the remaining capacity of arc $(i, j)$ in $G_{1}$.

The flow $f_{2}$ must go through arc $(i, j)$. Otherwise, because the only difference between $G_{1}^{\prime}$ and $G_{2}^{\prime}$ is capacity of arc $(i, j)$, if flow $f_{2}$ in $G_{2}^{\prime}$ does not go through arc $(i, j)$, there must also exist the same flow in resulted network $G_{1}^{\prime}$, which is a contradiction the fact that $\left(\mathbf{x}^{(1)}, \mathbf{y}^{(1)}\right)$ completely destroys network $G_{1}$. So 
flow $f_{2}$ must go through arc $(i, j)$. That is the remaining capacity of arc $(i, j)$ in $G_{2}^{\prime}, u_{i j}^{(2)}\left(1-y_{i j}^{(1)}\right)>0$, which lead to the remaining capacity of arc $(i, j)$ in $G_{1}^{\prime}$, $u_{i j}^{(1)}\left(1-y_{i j}^{(1)}\right)>0$.

The flow $f_{2}$ in $G_{2}$ consists of three parts: (1) flow from source node $s$ to node $i$; (2) flow on $\operatorname{arc}(i, j)$; (3) flow from node $j$ to node $t$. Let $f_{1}=\min \left\{f_{2}, u_{i j}^{(1)}\left(1-y_{i j}^{(1)}\right)\right\}$, as the capacities of all nodes/arcs in $G_{1}^{\prime}$, except for $(i, j)$, are the same as the corresponding ones in $G_{2}^{\prime}$ and $f_{1} \leq f_{2}$, there also exists $f_{1}$ amount of flow from node $s$ to node $i$ and node $j$ to node $t$ in $G_{1}^{\prime}$. Then there exists $f_{1}$ amount of flow from node $s$ to node $t$ in $G_{1}^{\prime}$, which is a contradiction to the fact that $R_{b}^{(1)}$ is critical budget $R_{b}$ for network $G_{1}$. Therefore $R_{b}^{(1)}<R_{b}^{(2)}$ cannot hold.

We have proved $R_{b}^{(1)}=R_{b}^{(2)}$ for $G_{1}$ and $G_{2}$, which have the same supply and demand amounts, and have only one $\operatorname{arc}(i, j)$ with different capacities. If $G_{1}$ and $G_{2}$ have $m(m \geq 2)$ arcs with different capacities, we can start from $G_{1}$ and change the capacity of only one arc so that the resulted network has $m-1$ arcs with different capacities with $G_{2}$. The resulted network must have same critical budget $R_{b}$ as $G_{1}$. Then based on this resulted network, we continue to change the capacity of only arc so that the resulted network has $m-2$ arcs with different capacities with $G_{2}$. Repeating this steps, we must have that the critical budgets of $G_{1}$ and $G_{2}$ are the same.

If $G_{1}$ and $G_{2}$ have different node capacities, their critical budgets $R_{b}$ should also be the same, because we can use Theorem 1 to split each node to one arc and repeat above procedure to prove $G_{1}$ and $G_{2}$ have the same critical budget $R_{b}$.

Therefore, under any changes on node/arc capacities, the critical budget $R_{b}$ for this network does not change.

From Theorem 2, Lemma 1 and Lemma 2, the critical budget $R_{b}$ is only related to the network topology $G=(N, A)$, the set of sources $S(k)$ and destinations $D(k)$ for all $k \in K$, and the interdiction costs $p_{i}, q_{i j}$ for all $i \in N,(i, j) \in A$, but this value $R_{b}$ is not related to supply and demand amounts and capacities of nodes and arcs. For example, in a simple graph in Fig. 2, $K=1$ and node $i$ is the source node and node $j$ is the destination node. Consider two cases: (1) $u_{i j}=20, q_{i j}=10$, (2) $u_{i j}=40, q_{i j}=10$. Critical budget $R_{b}$ is the budget which can just destroy the connection between $i$ and $j$. For case $1, R_{b}=q_{i j}=10$ and for case $2, R_{b}=q_{i j}=10$. Actually, no matter what value $u_{i j}$ is, the critical budget $R_{b}$ is always the arc's interdiction cost $q_{i j}$ because budget $R_{b}$ is about destroying the whole network. Also, it is easy to check that the supply and demand amounts have no influence on the critical budget $R_{b}$.

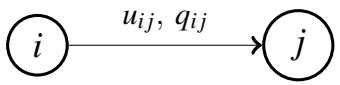

Fig. 2 An example for Lemma 2 
In the following, we present a formulation to estimate $R_{b}$ for both binary and continuous interdiction:

$$
\begin{aligned}
\max _{f, \mu} & \sum_{k \in K} \sum_{i \in D(k)} \mu_{i}^{t k} \\
\text { s.t. } & \sum_{j:(i, j) \in A} f_{i j}^{k}-\sum_{j:(j, i) \in A} f_{j i}^{k}= \begin{cases}\mu_{i}^{s k}, & i \in S(k), \\
-\mu_{i}^{t k}, & i \in D(k), \\
0, & i \notin\{S(k), D(k)\},\end{cases} \\
& \sum_{j:(i, j) \in A} \sum_{k} f_{i j}^{k} \leq p_{i}, \quad \forall i \in N \\
& \sum_{k \in K} f_{i j}^{k} \leq q_{i j}, \quad \forall(i, j) \in A \\
& f_{i j}^{k} \geq 0, \forall(i, j) \in A, k \in K
\end{aligned}
$$

where decision variables include $f_{i j}^{k}$ for all $(i, j) \in A, k \in K$ (vector denoted by $f$ ) and $\mu_{i}^{t k}$ for all $k \in K, i \in D(k)$ and $\mu_{i}^{s k}$ for all $k \in K, i \in S(k)$ (vector denoted by $\mu$ ). This formulation is to find a maximum flow in the network $G=(N, A)$ with node/arc capacity replaced by corresponding interdiction costs.

Theorem 3 For network $G=(N, A)$ with single commodity flow (i.e., $K=1)$, if source and destination nodes are distinct, the critical budget $R_{b}$ is equal to the optimal objective value of formulation (9).

Proof. By Theorem 1, the network can be transformed into a network $G^{\prime}=\left(N^{\prime}, A^{\prime}\right)$, with only arc capacity and arc interdiction.

As source and destination nodes are distinct, we can add another source node connected to all sources and also another destination connected to all destinations, which make the network become a single flow problem with single source and single destination. If the a budget can totally destroy the minimum cut, none of the demands in $D(1)$ can be satisfied. From Lemma 2, the critical budget $R_{b}$ is only related to the values of interdiction $\operatorname{cost} q_{i j}^{\prime}$ on each arc of $G^{\prime}$. Therefore, the formulation for finding minimum cut regarding the interdiction cost in $G^{\prime}$ can be used to find the upper bound of the critical budget $R_{b}$, and

$$
R_{b} \leq \min \sum_{(i, j) \in A^{\prime}: i \in N_{1}^{\prime}, j \in N_{2}^{\prime}} q_{i j}^{\prime}
$$

where $N_{1}^{\prime}, N_{2}^{\prime}$ is a partition of node set $N^{\prime}$ such that sources are in $N_{1}^{\prime}$ and destinations are in $N_{2}^{\prime}$. By taking duality of this minimum cut problem with strong duality property, the optimal objective value of formulation (9) in case of $K=1$ can be obtained to give an upper bound for $R_{b}$.

Now, we claim that the optimal objective value of formulation (9) gives an lower bound for $R_{b}$. In fact, let $G^{\prime \prime}$ be a network with same parameters as $G^{\prime}$ except the capacity $u_{i j}^{\prime \prime}=q_{i j}^{\prime}$ for each arc. The two networks $G^{\prime}$ and $G^{\prime \prime}$ have the same critical budget $R_{b}$, as proved by Lemma 2 . Next, we compute the critical budget $R_{b}$ of network $G^{\prime \prime}$. 
As destroying one unit of capacity on any arc can make at most one unit of demand unsatisfied. Assume that the maximum flow in the network $G^{\prime \prime}$ is $f_{\max }$ units under capacities $u_{i j}^{\prime \prime}$ 's. Therefore, at most $f_{\max }$ units of flow can be satisfied. The least number of units of capacities should be destroyed in the network is $f_{\max }$ in order to make none of demands be satisfied. For an $\operatorname{arc}(i, j) \in A^{\prime \prime}$, the corresponding cost for destroying one unit of capacity is $q_{i j}^{\prime} / u_{i j}^{\prime \prime}=1$. Assume the flow on this arc is $f_{i j}^{\prime \prime}$, and the corresponding cost to destroy this flow is f, bounded by $u_{i j}^{\prime \prime}=q_{i j}^{\prime}$. Therefore, in the problem of finding maximum flow in $G^{\prime \prime}$, assume that each arc has an associated cost $f_{i j}^{\prime \prime}$ bounded by $q_{i j}^{\prime}$, the objective of this problem gives the cost for destroying the maximum flow.

Now, by transforming the problem on $G^{\prime \prime}$ back to $G$ with node capacity, the optimal objective value of formulation (9) is the cost for destroy of the maximum flow, which gives an lower bound for $R_{b}$.

Therefore, as the optimal objective value of formulation (9) gives both lower and upper bound for $R_{b}$, it is equal to $R_{b}$.

Theorem 3 presents an approach for computing the critical budget $R_{b}$. However, this approach cannot be generalized to a multicommodity flow case, even if each type of commodity has only a single source and a single destination. For example, consider a similar case from [33] in the following Fig. 3. There are four types of commodities, each with one source node and one destination node. All arcs/nodes interdiction costs are fixed to 1 and all capacities of arcs and nodes are fixed to 1 . The critical budget $R_{b}$ is 2 and corresponding interdiction plan is removing two nonadjacent arcs. But the formulation (9) is to get the total maximum flow from each $s_{i}$ to each $t_{i}, i=1,2,3,4$ and the maximum flow is $4 / 3$, with flow from each $s_{i}$ to each $t_{i}, i=1,2,3,4$ to be $1 / 3$.

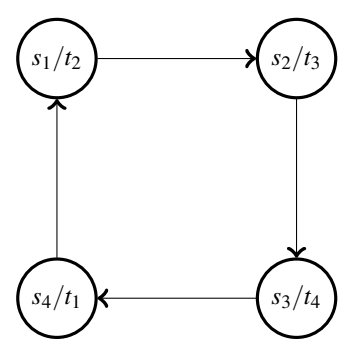

Fig. 3 maximum flow is less than $R_{b}$

In general multicommodity flow networks, the critical budget $R_{b}$ is related to the multicommodity maximum flow in the network. There have been considerable research on this, for example [34]. Therefore we only investigate the relation between the two. We have following two theorems to obtain the critical budget $R_{b}$. In the network $G=(N, A)$ with node capacity $v_{i}$ for any $i \in N$, arc capacity $u_{i j}$ for any $(i, j) \in A$, interdiction costs $p_{i}$ for all $i \in N$ and $q_{i j}$ for all $(i, j) \in A$, and sources $S(k)$ 
to destinations $D(k)$ for any $k \in K$, the disconnect set is union of a subset of node set of $N$ and a subset of arc set of $A$, whose removal will make none of the demands be satisfied. The minimum disconnect set is the one with minimum total capacity of vertices and arcs among all possible disconnect subsets. This can be considered as a direct generalization of $s-t$ minimum cut in a multicommodity flow network. Similarly, we define the minimum disconnect set regarding the interdiction cost as the one with minimum total interdiction cost of vertices and arcs among all possible disconnect subset, and this set can be found by the following formulation:

$$
\begin{array}{ll}
\min & \sum_{i \in A} p_{i} v_{i}+\sum_{(i, j) \in A} q_{i j} \xi_{i j} \\
\text { s.t. } & \sum_{i \in P_{i j}^{k}} v_{i}+\sum_{(i, j) \in P_{i j}^{k}} \xi_{i j} \geq 1, \forall P_{i j}^{k} \in \Omega_{i j}^{k}, k \in K, i \in S(k), j \in D(k) \\
& v_{i}, \xi_{i j} \in\{0,1\}, \forall i \in N,(i, j) \in A
\end{array}
$$

where $P_{i j}^{k}$ is a path from $i$ to $j$, and $\Omega_{i j}^{k}$ is the set of all possible paths from one source to one destination of type $k$. Both decision variables $v_{i}, \xi_{i j} \in\{0,1\}$ are defined to indicate whether node $i$ and arc $(i, j)$ are chosen into the disconnect set or not. The constraints in (10b) ensure that every path from $i$ to $j$ will be disconnected after removal of at least one node or one arc on this path.

Theorem 4 For the network $G=(N, A)$ with node capacity $v_{i}$ for any $i \in N$ and arc capacity $u_{i j}$ for any $(i, j) \in A$, the critical budget value $R_{b}$ under values $p_{i}, q_{i j}$, is equal to the optimal objective value of formulation (10).

Proof. If the a budget can totally destroy the disconnect set, none of the demands in $D(k)$ for any $k \in K$ can be satisfied. Now we consider the equivalent graph $G^{\prime}$ with only arc capacity and arc interdiction by Theorem 1 . From Lemma 2, the critical budget $R_{b}$ is only related to the values of interdiction cost $p_{i j}^{\prime}$ on each arc of $G^{\prime}$. Therefore, the formulation (10) for finding the minimum disconnect set consisting of only arcs regarding the interdiction cost in $G^{\prime}$ can be used to find the upper bound of the critical budget $R_{b}$, and

$$
R_{b} \leq \min \sum_{(i, j) \in A^{\prime \prime}} q_{i j}^{\prime}
$$

where $A^{\prime \prime}$ is a disconnect cut set such that in the graph $G^{\prime \prime}=\left(N^{\prime}, A^{\prime} \backslash A^{\prime \prime}\right)$, no demand of any type can be satisfied. Now transforming this graph to $G$ from $G^{\prime}$, we now have that the optimal objective value of formulation (10) presents an upper bound for $R_{b}$.

On the other hand, assume that one interdiction $(\mathbf{x}, \mathbf{y})$ is corresponding to critical budget $R_{b}$ that can make all demand be unsatisfied. By Theorem 2, we assume that this interdiction $(\mathbf{x}, \mathbf{y})$ is a binary interdiction on $G$. These removed nodes and arcs by $(\mathbf{x}, \mathbf{y})$ make none of the demands be satisfied, and by definition, the set formed by them is a disconnect set. Their corresponding value for interdiction $R_{b}$ should be larger than or equal to the disconnect set with minimum interdiction cost, that is $R_{b}$ is larger than or equal to the optimal objective value of formulation (10).

Therefore, the critical budget $R_{b}$ is equal to the optimal objective value of formulation (10). 
Theorem 4 presents an approach to compute the value for $R_{b}$. However, the formulation (10) requires to find all possible paths of different types of commodities from sources to destinations. In [33], a special case with single source and single destination has been considered, and cutting plane algorithm and branch-and-cut algorithm is used to find the minimum disconnect subset. For the same case, an implicit enumeration algorithm and an arc-chain algorithm are presented in [35]. Generally, when $K \geq 3$, this problem of finding minimum disconnect set is NP-complete (see [36]).

In the following, we present another approach which can give a lower bound for $R_{b}$ by solving a linear program. This is summarized in the following theorem and its proof is similar to the second part of the proof in Theorem 3.

Theorem 5 For the network $G=(N, A)$ with node capacity $v_{i}$ for any $i \in N$ and arc capacity $u_{i j}$ for any $(i, j) \in A$, the critical budget value $R_{b}$ under values $p_{i}, q_{i j}$, has a lower bound given by the optimal objective value of formulation (9).

\subsubsection{Critical budget $R_{a}$}

Theorem 6 In network $G=(N, A)$, with node capacity $v_{i}$ for all $i \in N$ and arc capacity for all arc $(i, j) \in A$, sources $S(k)$ and destinations $D(k)$ for all commodity $k \in K$, and supply amount $s_{i}^{k}$ for all $i \in S(k), k \in K$ and demand amount $d_{j}^{k}$ for all $j \in D(k), k \in K$, the critical budget value $R_{a}$ under the same interdiction costs $p_{i}, q_{i j}$ of continuous interdiction is less than or equal to the corresponding value of binary interdiction.

Proof. Let $R_{a}^{(b)}, R_{a}^{(c)}$ be the critical budget $R_{a}$ for network $G$ to be interdicted under binary and continuous interdictions, respectively.

Since $R_{a}^{(c)}$ is the critical budget, any continuous interdiction $\left(\mathbf{x}^{(c)}, \mathbf{y}^{(c)}\right) \in \mathscr{I}_{c}$ with this budget cannot make any demand in the resulted network be unsatisfied. A binary interdiction $\left(\mathbf{x}^{(b)}, \mathbf{y}^{(b)}\right) \in \mathscr{I}_{b}$ under budget $R_{a}^{(c)}$ is also feasible for continuous interdiction, i.e., $\left(\mathbf{x}^{(b)}, \mathbf{y}^{(b)}\right) \in \mathscr{I}_{c}$. Thus, any binary interdiction $\left(\mathbf{x}^{(b)}, \mathbf{y}^{(b)}\right)$ also cannot make any demand in the resulted network be unsatisfied.

The critical budget $R_{a}$ is defined as the largest budget that all demand in the resulted network are still satisfied after interdiction. Therefore, the inequality $R_{a}^{(c)} \leq$ $R_{a}^{(b)}$ holds.

In network $G=(N, A)$ with sources $S(k)$ and destinations $D(k)$ for any commodity $k \in K$, let

$$
\mathscr{Z}=\left\{\mathbf{z} \in \mathbb{R}^{|A| \times K}: \text { constraints }(1 \mathrm{~b})-(1 \mathrm{e})\right\},
$$

where $\mathbf{z}$ is a vector consisting of $z_{i j}^{k}$, for any $(i, j) \in A, k \in K$, denote all feasible flows in network $G$. The residual network $G^{z}=(N, A)$ corresponding to a flow $\mathbf{z} \in \mathscr{Z}$ has node capacity $v_{i}^{z}=v_{i}-\sum_{j:(i, j) \in A} \sum_{k} z_{i j}^{k}$ for any $i \in N$ and arc capacity $u_{i j}^{z}=u_{i j}-\sum_{k} z_{i j}^{k}$ for any $(i, j) \in A$. 
For each type $k \in K$ commodity and each destination node $t \in D(k)$, let $\rho^{k t}$ be a vector consisting of flows $\rho_{i j}^{(k t)}$ on all arc $(i, j) \in A$, supply $\hat{s}_{i}^{(k t)}$ for all $i \in S(k)$ (vector denoted by $\left.\hat{S}^{(k t)}\right)$ and demand $\hat{d}^{(k t)}$, under the case of type $k$ commodity and one destination $t \in D(k)$ in the residual network of flow $\mathbf{z}$. Therefore, $\rho^{(k t)}$ is limited by the following constraints in the set

$$
\left.\begin{array}{rl}
\mathscr{P}(\mathbf{z}, k, t) & \left.\left\{\begin{array}{l}
\sum_{j:(i, j) \in A} \rho_{i j}^{(k t)}-\sum_{j:(j, i) \in A} \rho_{j i}^{(k t)}= \begin{cases}\hat{s}_{i}^{(k t)}, & i \in S(k) \\
-\hat{d}^{(k t)}, & \\
0, & i \notin\{S(k), t\}, \forall i \in N \\
\sum_{j:(i, j) \in A} \rho_{i j}^{(k t)} \leq v_{i}^{z}, \forall i \in N \\
0 \leq \rho_{i j}^{(k t)} \leq u_{i j}^{z}, \forall(i, j) \in A \\
\hat{s}_{i}^{(k t)} \geq 0, \forall i \in S(k) \\
\hat{d}^{(k t)} \geq 0\end{cases}
\end{array}\right\} . \hat{d}^{(k t)}\right) \in \mathbb{R}^{|A|+|S(k)|+1}
\end{array}\right\} .
$$

Theorem 7 In network $G=(N, A)$, if the interdiction cost $p_{i}=v_{i}$ for any $i \in N$ and $q_{i j}=u_{i j}$ for any $(i, j) \in A$, the critical budget $R_{a}$ for continuous interdiction has a lower bound given by the optimal objective value of the following problem:

$$
\max _{\mathbf{z} \in \mathscr{Z}} \min _{k \in K, t \in D(k)} \max _{\left(\rho^{(k t)}, \hat{s}^{(k t)}, \hat{d}^{(k t)}\right) \in \mathscr{P}(\mathbf{z}, k, t)} \hat{d}^{(k t)}
$$

In formulation (11), under a feasible flow $\mathbf{z}$ to satisfy all demands, in the residual network of this flow $\mathbf{z}$, for each type $k \in K$ commodity and each destination node $t \in D(k)$, a maximum flow is to be determined from all possible sources $i \in S(k)$. When $p_{i}=v_{i}, q_{i j}=u_{i j}$ for any $i \in N,(i, j) \in A$, one unit of budget can destroy one unit of capacity, or finally at most one unit of commodity demand. Therefore, a budget $R^{(k t)}$ that can make demand at node $t \in D(k)$ be unsatisfied must be at least equal to maximum flow. Then through all types of commodities and all destination nodes, the minimum of $R^{(k t)}$ 's for any $k \in K$ and $t \in D(k)$ determines a budget $R^{z}$ under flow $\mathbf{z}$ such that any additional budget on $R^{z}$ will make some destination's demand be unsatisfied. Consider all possible flows $\mathbf{z} \in \mathscr{Z}$ to satisfy all original demands, the maximum budget of $R^{z}$ 's is related to the critical budget $R_{a}$. In the following, we formally prove this theorem.

Proof. Assume the optimal objective value to formulation (11) is $R_{a}^{*}$. We claim $R_{a} \geq$ $R_{a}^{*}$, otherwise $R_{a}<R_{a}^{*}$, which implies that with budget $R_{a}+\varepsilon\left(0<\varepsilon<R_{a}^{*}-R_{a}\right)$, by definition of $R_{a}$, there exists an interdiction such that there is one commodity whose demand cannot be totally satisfied in resulted network.

As $p_{i}=v_{i}, q_{i j}=u_{i j}$ for any $i \in N,(i, j) \in A$, one unit of budget can destroy one unit of capacity, or finally at most one unit of commodity demand. Because $R_{a}^{*}$ is the optimal solution to formulation (11), which implies at least $R_{a}^{*}$ amount of budget is needed in order to make some commodity's demand unsatisfied, which is a contradiction to the fact that budget $R_{a}+\varepsilon$ can make some commodity's demand unsatisfied. Therefore we have $R_{a} \geq R_{a}^{*}$. 
The formulation (11) is a tri-level optimization problem. To solve this problem, we introduce $\eta=\min _{k \in K, t \in D(k)} \max _{\left(\rho^{(k t)}, \hat{s}^{(k t)}, \hat{d}^{(k t)}\right) \in \mathscr{P}(\mathbf{z}, k, t)} \hat{d}^{(k t)}$. Then the formulation (11) is equivalent to the following linear program:

$$
\begin{aligned}
\max _{\mathbf{z} \in \mathscr{Z}} & \eta \\
\text { s.t. } & \eta \leq \hat{d}^{(k t)}, \quad \forall t \in D(k), k \in K \\
& \left(\rho^{(k t)}, \hat{s}^{(k t)}, \hat{d}^{(k t)}\right) \in \mathscr{P}(\mathbf{z}, k, t), \quad \forall t \in D(k), k \in K
\end{aligned}
$$

In Theorem 7, we assume that $p_{i}=v_{i}$ for any $i \in N$ and $q_{i j}=u_{i j}$ for any $(i, j) \in A$. That is, it takes one unit budget to interdict one unit of capacity. If $p_{i}=C v_{i}$ and $q_{i j}=C u_{i j}$, where $C$ is a positive constant, the lower bounded for $R_{a}$ is the optimal objective value of (11), multiplied by $C$.

The formulation (11) can find the lower bound for $R_{a}$, but it cannot obtain the value for $R_{a}$ in a multicommodity network directly. For example, in Fig. 4, there are three types of commodities, and each one has single source $s_{k}$ and destination $t_{k}$ $(k=1,2,3)$ with supply amount as 1 on each $s_{i}$ and the demand as 1 on each $t_{j}$. The capacities for 4 arcs are given in this figure, and all other arcs have capacity as 100 . We consider only arc interdiction, and the interdiction cost for each arc is equal to its capacity. Assume the amount of flow on arc $\left(s_{1}, t_{1}\right)$ of commodity 1 is $z_{1}$, on arc $\left(i_{1}, j_{1}\right)$ of commodity 2 is $z_{2}$ and on arc $\left(s_{3}, t_{3}\right)$ of commodity 3 is $z_{3}$. Thus to satisfy all demands, the flow of commodity 1 on arc $\left(i_{1}, j_{1}\right)$ is $1-z_{1}$, and commodity 2 on $\left(i_{2}, j_{2}\right)$ is $1-z_{2}$, and commodity 3 on $\left(i_{2}, j_{2}\right)$ is $1-z_{3}$. Then in the residual network, additional maximum flow of commodity 1 is $2-z_{1}+\left(2-\left(1-z_{1}\right)-z_{2}\right)=3-z_{2}$, additional maximum flow of commodity 3 is $2-z_{3}+\left(2-\left(1-z_{2}\right)-\left(1-z_{3}\right)\right)=2+z_{2}$. Thus the optimal objective value of formulation (11) is at $\operatorname{most}_{\max } \max _{0 \leq 1} \leq \min \{3-$ $\left.z_{2}, 2+z_{2}\right\}=2.5$. However, the critical budget $R_{a}$ is 3 . Therefore, formulation (11) can only obtain a lower bound for $R_{a}$.

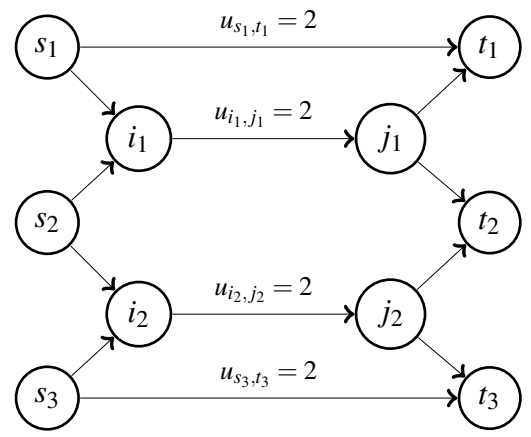

Fig. 4 Example for critical budget $R_{a}$

However, for a single commodity flow problem, we have the following corollary to obtain critical budget $R_{a}$ in continuous interdiction case. 
Corollary 1 For network $G=(N, A)$ with single commodity flow with single destination node, if $p_{i}=v_{i}, q_{i j}=u_{i j}$ for any $i \in N,(i, j) \in A$, the critical budget $R_{a}$ for continuous interdiction is equal to the optimal objective value of formulation (11).

Proof. When $K=1$ and there is only one destination node, optimal value of formulation (11) $R_{a}^{*}$ becomes the maximum flow from source nodes to destination node minus the demand of the commodity, that is the minimum cut of the network is $R_{a}^{*}+\sum_{i \in D(k)} d_{i}$. Because $p_{i}=v_{i}, q_{i j}=u_{i j}$ for any $i \in N,(i, j) \in A$, we can use budget $R>R_{a}^{*}$ to destroy any $R$ amount of capacity from the minimum cut and the resulted network cannot make all demands be satisfied. Thus $R_{a}^{*}$ is an upper bound of critical budget $R_{a}$. From Theorem $7, R_{a}^{*}$ is a lower bound of critical budget $R_{a}$. Therefore, $R_{a}^{*}=R_{a}$.

The formulation (11) cannot apply to the case for general values of $p_{i}, q_{i j}$, even for single commodity with single destination network. For example, in Fig. 5, we show an example with single-commodity flow with one source $s$ with supply amount 2 and one destination $t$ with demand amount 2. Consider continuous interdiction on only arcs, the critical budget $R_{a}$ is 1 , and the corresponding interdiction plan is to remove arc $\left(i_{3}, t\right)$. However, the optimal objective value to $(11)$ is 2 , with corresponding solution as $z_{i_{1}, t}=z_{i_{2}, t}=0, z_{i_{3}, t}=2$, in whose residual network, we can still send 2 units of flow via arc $\left(i_{1}, t\right)$ and $\left(i_{2}, t\right)$.

The formulation (11) cannot apply to binary interdiction case. For example, in Fig. 5, if we change the interdiction cost of arc $\left(i_{3}, t\right)$ to $q_{i_{3}, t}=2$, then $p_{i}=v_{i}, q_{i j}=u_{i j}$ holds for the network. Assume the supply amount and demand amount are both 1.5, then for binary interdiction, the critical budget $R_{a}=3$, and the corresponding interdiction plan is to remove arc $\left(i_{3}, t\right)$ and $\left(i_{2}, t\right)$. But the optimal value of formulation (11) is 1.5 . Therefore, formulation (11) cannot be used to obtain critical budget $R_{a}$ for binary interdiction.

Different from $R_{b}$, the critical budget $R_{a}$ is related to parameters $s_{i}^{k}, d_{j}^{k}, v_{i}, u_{i j}, p_{i}, q_{i j}$ of the network. For example, in Fig. 5, if the supply and demand amounts are 1, critical budget $R_{a}$ will be 2 . Also, different $v_{i}$ and $u_{i j}$ can change the value of $R_{a}$. For example, in Fig. 5, if $u_{i_{1}, t}=u_{i_{2}, t}=2$, critical budget $R_{a}$ will increase to 2. Additionally, if $q_{i_{1}, t}=q_{i_{2}, t}=q_{i_{3}, t}=2, R_{a}$ will be 2 .

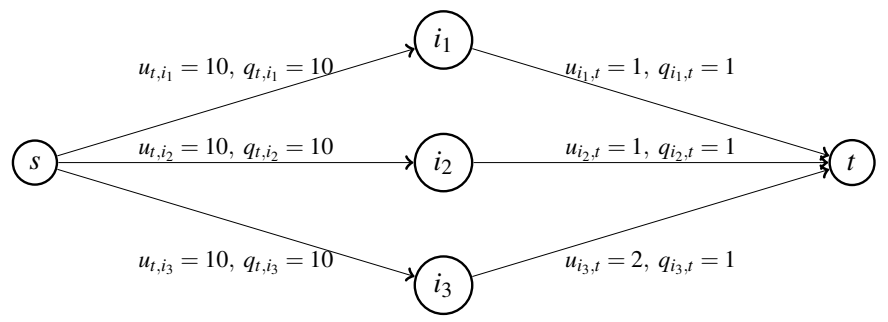

Fig. 5 Example for critical budget $R_{a}$ 


\subsection{Effects of budget increasing}

\subsubsection{Marginal revenue for continuous interdiction}

For continuous interdiction, we define the marginal revenue at $R$ as the increased amount of unsatisfied demands that the interdictor can create by increasing budget by 1 , where we still assume that the operator always tries to minimize the unsatisfied demand under any interdiction.

For example, in Fig. 1(b), the marginal revenue for budget $R=4.4$ is positive, and thus the total unsatisfied demands increase as $R$ increases; while for budget $R=$ 6.4 , the marginal revenue is 0 and the total unsatisfied demands stay the same even interdiction budget increases until 7.2. Therefore for the interdictor with budget $R=$ 6.4 , it is not necessary to increase the budget unless the budget can increase to more than 7.2 .

Define the sum set $\mathscr{D}$ of demand amount set $\left\{d_{j}^{k}: \forall k \in K, j \in D(k)\right\}$ as the set of different summation values of all possible subsets of $\left\{d_{j}^{k}: \forall k \in K, j \in D(k)\right\}$. That is, the sum set $\mathscr{D}$ consists of all possible values of $d_{i_{1}}+d_{i_{2}}+\cdots+d_{i_{t}}$, where $\left\{d_{i_{1}}, d_{i_{2}}, \cdots, d_{i_{t}}\right\}$ is a subset of $\left\{d_{j}^{k}: \forall k \in K, j \in D(k)\right\}$. Note that 0 is always in the sum set.

Theorem 8 In continuous interdiction on network $G=(N, A)$, iffor some budget $R \geq$ $0, F(R)$ is not in sum set $\mathscr{D}$ of demand set $\left\{d_{j}^{k}: \forall k \in K, j \in D(k)\right\}$, the corresponding marginal revenue at $R$ is strictly larger than 0 .

Proof. When the budget $R \geq 0$, because $F(R)$ is not in sum set $\mathscr{D}$ of demand set $\left\{d_{j}^{k}: \forall k \in K, j \in D(k)\right\}$, at least at one destination node, a certain commodity $k$ 's demand is partially unsatisfied. We assume that the partially unsatisfied amount $\lambda_{j}^{k}$ is on the demand $d_{j}^{k}$. By "partially unsatisfication", we mean that $0<\lambda_{j}^{k}<d_{j}^{k}$ for some $k \in K, j \in D(k)$.

As node capacity and node interdiction can be transformed into corresponding arc capacity and arc interdiction by Theorem 1, we first only consider the case for arcs. This theorem can be implied by the following claim: if budget $R$ makes the demand $d_{j}^{k}$ partially unsatisfied, then on any path $P$ from $S(k)$ to $j \in D(k)$ in the resulted network, there exists at least one arc, whose additional loss of partial capacity or complete removal strictly increases $F(R)$.

Assume the solution to $\mathbf{U D}(R)$ for $(\mathbf{x}, \mathbf{y}, \mathbf{z})$ is $\left(\mathbf{x}^{0}, \mathbf{y}^{0}, \mathbf{z}^{0}\right)$, with optimal objective value $\Lambda^{*}$. Let $\overline{\Delta R}$ be the minimum budget that could decrease the capacity of one arc on path $P$, to 0 , in addition to current interdiction $\mathbf{y}^{0}$. In the resulted network by $R$, the extra $\overline{\Delta R}$ can additionally remove the remainder capacity of one arc in path $P$. Then if we can prove that for any $\Delta R \in(0, \overline{\Delta R}), F(R+\Delta R)>F(R)$, our claim is true. In fact, we can prove it by the following contradiction approach.

Note $F(R)$ is monotonically increasing, then by contradiction, we have $F(R+$ $\Delta R)=F(R)=\Lambda^{*}$ if the above statement is not true, which means decrease of capacity of any arc limited by the additional budget $\Delta R$ can not increase $F(R)$.

Denote the path $P$ by $P=\left(e_{1}, e_{2}, \cdots, e_{m}\right)$ from some node in $S(k)$ to $j \in D(k)$ in the resulted network $G(k)$ by interdiction $\mathbf{y}^{0}$, consisting of $m \operatorname{arcs} e_{1}, e_{2}, \cdots, e_{m}$. Then 
from $G(k)$ we continue decreasing capacity, limited by the additional budget $\Delta R$, from the first arc $e_{1}$ in $P$. By this process we actually fix the outer level of $\mathbf{U D}(R)$, and then we call an oracle to solve the inner level of $\mathbf{U D}(R)$. Denote the optimal solution by $\left(\mathbf{y}^{1}, \mathbf{z}^{1}, \lambda^{1}, \Lambda^{1}\right)=\left\{y_{i j}^{1}, z_{i j}^{k, 1}, \lambda_{i}^{k, 1}, \Lambda_{k}^{1}: \forall i \in D(k), \forall(i, j) \in A, \forall k \in K\right\}$, where $\Lambda_{k}^{1}$ is the unsatisfied amount of type $k$ commodity, and $y_{i j}^{1}=y_{i j}^{0}$ for all $(i, j) \in A$ except $y_{e_{1}}^{1}=y_{e_{1}}^{0}+\frac{\Delta R}{q_{e_{1}}}$. Thus, we have $\sum_{k} z_{e_{1}}^{k, 1} \leq u_{e_{1}}\left(1-y_{e_{1}}^{1}\right)<u_{e_{1}}\left(1-y_{e_{1}}^{0}\right)$, and then $\Lambda^{1} \geq$ $F(R)=\Lambda^{*}$. As explained above, we have similar analysis for node capacity and node interdiction by Theorem 1 , and thus we assume $\mathbf{x}^{1}$ is the corresponding solution for node interdiction.

Then we repeat this procedure separately on each arc of $e_{2}, \cdots, e_{m}$ on this path $P$ and obtain corresponding solutions $\left(\mathbf{x}^{2}, \mathbf{y}^{2}, \mathbf{z}^{2}, \lambda^{2}, \Lambda^{2}\right), \cdots,\left(\mathbf{x}^{m}, \mathbf{y}^{m}, \mathbf{z}^{m}, \lambda^{m}, \Lambda^{m}\right)$. As we just explained, we have $\sum_{k} z_{e_{i}}^{k, i}<u_{e_{i}}\left(1-y_{e_{i}}^{0}\right)$ and $\Lambda^{i} \geq \Lambda^{*}$, for any $i=1, \cdots, m$; on the other hand, $F(R+\Delta R)$ is the optimal value for $\mathbf{U D}(R+\Delta R), \Lambda^{i} \leq F(R+\Delta R)=$ $\Lambda^{*}$ (second equality from the assumption of contradiction), for any $i=1, \cdots, m$. Thus, $\Lambda^{i}=\Lambda^{*}$, for any $i=1, \cdots, m$.

Denote $\left(\sum_{l=1}^{m} \mathbf{z}^{l} / m, \sum_{l=1}^{m} \lambda^{l} / m, \sum_{l=1}^{m} \Lambda^{l} / m\right)$ by $(\overline{\mathbf{z}}, \bar{\lambda}, \bar{\Lambda})$. Then $\bar{\Lambda}=\sum_{l=1}^{m} \Lambda^{l} / m=$ $\Lambda^{*}$. Now we will show: (a) $\left(\mathbf{x}^{0}, \mathbf{y}^{0}, \overline{\mathbf{z}}, \bar{\lambda}, \bar{\Lambda}\right)$ is feasible to $\mathbf{U D}(R)$, but (b) $\left(\mathbf{x}^{0}, \mathbf{y}^{0}, \overline{\mathbf{z}}, \bar{\lambda}, \bar{\Lambda}\right)$ is not optimal to $\mathbf{U D}(R)$.

(a) Feasibility of $\left(\mathbf{x}^{0}, \mathbf{y}^{0}, \overline{\mathbf{z}}, \bar{\lambda}, \bar{\Lambda}\right)$ to $\mathbf{U D}(R)$. Each $\left(\mathbf{x}^{0}, \mathbf{y}^{0}, \mathbf{z}^{l}, \lambda^{l}, \Lambda^{l}\right), l=1, \cdots, m$, is feasible to the constraints in formulation (8), as $v_{i}\left(1-x_{i}^{0}\right) \leq v_{i}\left(1-x_{i}^{1}\right)$ and $u_{i j}(1-$ $\left.y_{i j}^{0}\right) \leq u_{i j}\left(1-y_{i j}^{1}\right)$. Thus, $\left(\mathbf{x}^{0}, \mathbf{y}^{0}, \overline{\mathbf{z}}, \bar{\lambda}, \bar{\Lambda}\right)$ is feasible to $\mathbf{U D}(R)$.

(b) Non-optimality of $\left(\mathbf{x}^{0}, \mathbf{y}^{0}, \overline{\mathbf{z}}, \bar{\lambda}, \bar{\Lambda}\right)$ to $\mathbf{U D}(R)$. For each $e_{r} \in P$,

$$
\begin{aligned}
\bar{z}_{e_{r}} & =\sum_{l=1}^{m} \sum_{k \in K} z_{e_{r}}^{k, l} / m=\sum_{l=1, l \neq r}^{m} \sum_{k \in K} z_{e_{r}}^{k, l} / m+\sum_{k \in K} z_{e_{r}}^{k, r} / m \\
& <\sum_{l=1, l \neq r}^{m} u_{e_{l}}\left(1-x_{e_{l}}^{0}\right) / m+u_{e_{r}}\left(1-x_{e_{r}}^{0}\right) / m<u_{e_{r}}\left(1-x_{e_{r}}^{0}\right),
\end{aligned}
$$

where the inequality is implied by $\sum_{k} z_{e_{i}}^{k, r}<u_{e_{r}}\left(1-x_{e_{r}}^{0}\right), r=1, \ldots, m$. This inequality shows the residual capacity of $\overline{\mathbf{z}}$, and the residual capacity for node is similar. Thus on path $P$ we can still send flow from source node to destination node. That is, if network is interdicted by $\left(\mathbf{x}^{0}, \mathbf{y}^{0}\right)$, we can send more than flow $\bar{z}$ for commodity $k$. Thus, the total unsatisfied demand after interdiction $\left(\mathbf{x}^{0}, \mathbf{y}^{0}\right)$ should be less than $\bar{\Lambda}=\Lambda^{*}$, and this implies that $\Lambda^{*}$ is not optimal to $\mathbf{U D}(R)$.

Thus, $F(R)<\Lambda^{*}$, which is a contradiction to our assumption. Therefore, $F(R+$ $\Delta R)>F(R)$ is true, and this leads to our conclusion that marginal revenue is strictly larger than 0 .

This proof also shows the continuity of $F(R)$. In continuous interdiction, for two budgets $R_{1}, R_{2} \geq 0$, if for any $R \in\left[R_{1}, R_{2}\right], F(R)=F\left(R_{1}\right)=F\left(R_{2}\right)$, the curve of $F(R)$ and budget $R$ between $R_{1}$ and $R_{2}$ is called a plateau. For example, the curve in range $[6,7]$ of Fig. 1(b) is a plateau. Now we present a corollary regarding the number of plateaus in the curve of unsatisfied demand $F(R)$ with respect to $R$. Let $|\mathscr{D}|$ denote 
the number of elements in sum set $\mathscr{D}$ of demand set $\left\{d_{j}^{k}: \forall k \in K, j \in D(k)\right\}$. It is bounded by $2^{n}$, where $n=\sum_{k \in K}|D(k)|$ and $|D(k)|$ denotes the number of destination nodes for commodity $k \in K$.

Corollary 2 In continuous interdiction, the number of plateaus in the curve of unsatisfied demand $F(R)$ is at most $|\mathscr{D}|$.

Proof. From Theorem 8, we have that in case of continuous interdiction, if for some budget $R, F(R)$ is not in sum set $\mathscr{D}$ of demand set $\left\{d_{j}^{k}: \forall k \in K, j \in D(k)\right\}, F(R)$ will increase if there is additional interdiction budget besides $R$ until that $F(R)$ increases to a value in $\mathscr{D}$. So the only case that $F(R)$ stays unchanged is $F(R) \in \mathscr{D}$. Then the maximum number of plateaus in the curve of $F(R)$ and budget $R$ is at most the number of elements in $\mathscr{D}$, bounded by $2^{n}$.

\subsubsection{Effects of budget increasing for binary interdiction}

Theorem 9 In binary interdiction on network $G=(N, A)$, for some budget $R \geq 0$, if the corresponding unsatisfied demand $F(R)$ is strictly increasing, $R$ must be in sum set of set $\left\{p_{i}: \forall i \in N\right\} \cup\left\{q_{i j}: \forall(i, j) \in A\right\}$.

Proof. Any two interdictions, with different budgets strictly between two adjacent elements in ordered sum set of set $\left\{p_{i}: \forall i \in N\right\} \cup\left\{q_{i j}: \forall(i, j) \in A\right\}$, are the same, since no partial interdiction on arcs or nodes is allowed in this model. Thus, the objective for (8) for these two budgets are the same.

From Theorem 9, we have the following corollary. By Theorem 9 and this corollary, we have proved the Proposition 2(iv) for binary interdiction case.

Corollary 3 In binary interdiction on network $G=(N, A)$, for some budget $R \geq 0$, if $R$ is not in sum set of set $\left\{p_{i}: \forall i \in N\right\} \cup\left\{q_{i j}: \forall(i, j) \in A\right\}$, the unsatisfied demand $F(R)$ is equal to $F(\underline{R})$, where $\underline{R}$ is the largest element in the sum set with $\underline{R}<R$.

Theorem 10 In case of binary interdiction, denote $C=\max \left\{p_{i}: \forall i \in N\right\} \cup\left\{q_{i j}\right.$ : $\forall(i, j) \in A\}$, for some budget $R \geq 0$, if $F(R)$ is not in sum set $\mathscr{D}$ of demand set $\left\{d_{j}^{k}\right.$ : $\forall k \in K, j \in D(k)\}, F(R+C)>F(R)$.

The idea of this proof is similar to the continuous case in Theorem 8 , by changing $\Delta R=C$. If $p_{i}=q_{i j}=C$, for all $i \in N,(i, j) \in A$ and $C$ is a positive constant, for some budget $R \geq 0$, if $F(R)$ is not in sum set $\mathscr{D}$ of demand set $\left\{d_{j}^{k}: \forall k \in K, j \in D(k)\right\}$, $F(R+C)>F(R)$.

\section{Numerical Experiments}

In this section, all MIP formulations and algorithms are implemented in C++ using CPLEX 12.3 via IBM's Concert Technology library, version 2.9. All experiments 
were performed on a Linux workstation with 4 Intel(R) Core(TM)2 CPU $2.40 \mathrm{GHz}$ processors and 4GB RAM.

In the following, we test proposed approaches to find values for critical budgets $R_{a}, R_{b}$. All 12 networks for multicommodity flows used in the following experiments are modified based on test cases of C+ instances in [37]. For the costs and capacities on arcs and nodes, and supply and demand amounts of commodities, we modified their values with less types of commodities.

\subsection{Numerical experiment for critical budgets}

As discussed in Section 3.2, the critical budget $R_{a}$ can be computed by formulations (2) and (8), and theorems in Section 3.2.2 for different conditions, while $R_{b}$ can be computed by formulation (8) and theorems in Section 3.2.1.

In this section we first perform numerical experiment for solving formulation (2) under continuous interdiction by using techniques proposed in (B.4). We randomly generate interdiction costs $p_{i}$ for each node between 10 and 40 , and $q_{i j}$ for each arc between 4 to 20, and use formulation (2) in Section 2.2 to calculate the minimum-cost flow under interdiction budgets.

We test 12 different networks by gradually increasing budget $R$ from 0 (see Table 1). If the optimal solution is an abnormally large number, which is larger than $\sum_{i \in N} \max _{(i, j) \in A} c_{i j} \sum_{k \in K} \sum_{i \in D(k)} d_{i}^{k}$, it will be the budget with which there exists no optimal flow satisfying all demands, and thus the dual problem of inner problem of (B.4) is unbounded. By checking the optimal results, we can find the budget $R_{a}$, which is the budget from it some demand starts to become unsatisfied.

Table 1 shows that the optimal solution to formulation (2) for continuous interdiction with different interdiction budgets, and also the critical budget $R_{a}$ in each network. From the table we can see the optimal solution for continuous interdiction increases as budget increases. When the interdiction budget is large enough to make the problem infeasible, the optimal result will grow abnormally large and we use "-." and the followed empty spaces to denote it.

To test the formulation (11) in Theorem 7 for obtaining lower bound for critical budget $R_{a}$ when $p_{i}=v_{i}, q_{i j}=u_{i j}$ and Theorem 5 for $R_{b}$, we compute the critical budgets $R_{a}, R_{b}$. The results are shown in Table 2, where each network has $K$ source nodes and $K$ destination nodes, one for each type of commodity. For the values of $R_{a}$, we have verified the results obtained by the approach in Theorem 7, and also the method mentioned in Table 1.

In Table 1 and Table 2, comparing cases with same number of nodes and commodities but different numbers of arcs, we can find if the number of arcs is reducing, $R_{a}$ is also reduced. This is because when this number is reduced, the network becomes sparser and less vulnerable, the interdictor needs less budget to make commodities' demands unsatisfied. Critical budget $R_{b}$ also changes when the number of commodities is reduced in a fixed network. We can compare cases where the number of arcs and nodes remains the same, if the number of commodities decreases, $R_{a}$ increases. This is because when the number of commodities is reduced, there are more optional paths for commodities' flows. 
Table 1 Optimal solution for continuous interdiction with different budgets

\begin{tabular}{|c|c|c|c|c|c|c|c|c|c|c|c|c|}
\hline \multirow{3}{*}{$\begin{array}{r}|N| \\
|A| \\
K\end{array}$} & \multicolumn{9}{|c|}{8} & \multirow{2}{*}{\multicolumn{3}{|c|}{$\begin{array}{l}10 \\
60 \\
\end{array}$}} \\
\hline & \multicolumn{3}{|c|}{30} & \multicolumn{3}{|c|}{40} & \multicolumn{3}{|c|}{50} & & & \\
\hline & 3 & 4 & 6 & 3 & 4 & 6 & 3 & 4 & 6 & 3 & 4 & 6 \\
\hline$R=0$ & 316.0 & 452.0 & 561.0 & 316.0 & 436.0 & 542.0 & 222.0 & 306.0 & 405.0 & 357.0 & 465.0 & 609.0 \\
\hline 1 & 321.0 & 457.0 & 587.3 & 321.0 & 441.0 & 554.7 & 222.0 & 306.0 & 416.0 & 358.8 & 469.3 & 610.8 \\
\hline 2 & 326.0 & 462.0 & 614.7 & 326.0 & 446.0 & 569.6 & 222.0 & 306.0 & 427.9 & 361.3 & 469.3 & 636.3 \\
\hline 3 & 334.0 & 472.0 & 647.3 & 334.0 & 454.0 & 590.4 & 229.0 & 313.0 & 442.7 & 364.0 & 472.0 & 651.2 \\
\hline 4 & 362.0 & 500.3 & 682.7 & 342.0 & 462.0 & 616.7 & 236.0 & 320.0 & 463.3 & 364.0 & 472.0 & 674.6 \\
\hline 5 & 395.3 & 534.3 & - & 350.0 & 470.0 & 644.1 & 243.0 & 327.0 & 483.9 & 374.5 & 482.5 & 710.0 \\
\hline 6 & 434.0 & 573.0 & & 358.0 & 478.0 & 673.3 & 250.0 & 334.0 & 504.4 & 385.0 & 493.0 & 728.7 \\
\hline 7 & 439.0 & 578.0 & & 363.0 & 483.0 & - & 257.0 & 344.5 & - & 395.5 & 503.5 & 747.3 \\
\hline 8 & 444.0 & 583.0 & & 368.0 & 488.0 & & 264.0 & 355.0 & & 406.0 & 514.0 & 782.0 \\
\hline 9 & - & - & & 375.0 & 495.0 & & 264.0 & 356.0 & & 406.0 & 516.0 & 787.8 \\
\hline 10 & & & & 382.0 & 502.0 & & 268.0 & 360.0 & & 420.8 & 528.8 & 794.6 \\
\hline 11 & & & & 390.0 & 510.0 & & 272.0 & 365.0 & & 450.0 & 558.0 & 804.9 \\
\hline 12 & & & & 398.0 & 518.0 & & 276.0 & 373.0 & & 472.4 & 580.4 & 815.1 \\
\hline 13 & & & & 403.0 & 523.0 & & 280.0 & 384.0 & & 494.8 & 602.8 & 829.3 \\
\hline 14 & & & & 411.0 & 531.0 & & 288.0 & 395.0 & & 517.2 & 625.2 & 851.1 \\
\hline 15 & & & & 420.5 & 540.5 & & 288.0 & 406.0 & & 543.8 & 651.8 & 873.0 \\
\hline 16 & & & & 432.8 & 552.8 & & 291.3 & 417.0 & & - & - & - \\
\hline 17 & & & & 445.0 & 565.0 & & 298.0 & 420.6 & & & & \\
\hline 18 & & & & 453.7 & 577.0 & & 303.0 & 425.7 & & & & \\
\hline 19 & & & & - & - & & 308.0 & 431.0 & & & & \\
\hline 20 & & & & & & & 313.0 & 436.3 & & & & \\
\hline 21 & & & & & & & 318.0 & 441.7 & & & & \\
\hline 22 & & & & & & & 323.0 & 447.0 & & & & \\
\hline 23 & & & & & & & - & - & & & & \\
\hline$R_{a}$ & 8 & 8 & 4 & 18 & 18 & 6 & 22 & 22 & 6 & 15 & 15 & 15 \\
\hline
\end{tabular}

Table 2 Critical budget $R_{a}$ for continuous interdiction when $p_{i}=v_{i}, q_{i j}=u_{i j}$

\begin{tabular}{|c|c|c|c|c|c|c|c|c|c|c|c|c|}
\hline||$N \mid$ & \multicolumn{9}{|c|}{8} & \multirow{2}{*}{\multicolumn{3}{|c|}{$\begin{array}{l}10 \\
60\end{array}$}} \\
\hline$|A|$ & \multicolumn{3}{|c|}{30} & \multicolumn{3}{|c|}{40} & \multicolumn{3}{|c|}{50} & & & \\
\hline$K$ & 3 & 4 & 6 & 3 & 4 & 6 & 3 & 4 & 6 & 3 & 4 & 6 \\
\hline$R_{a}$ & 10 & 10 & 7 & 30 & 30 & 10 & 46 & 46 & 10 & 26 & 25 & 25 \\
\hline$R_{b}$ & 64 & 74 & 98 & 101 & 124 & 149 & 119 & 178 & 202 & 110 & 127 & 182 \\
\hline
\end{tabular}

Next, we perform numerical experiment for computing critical budgets for binary interdiction. First, we use formulation (8) to calculate critical budget $R_{b}$ by gradually increasing budget from 0 and check their corresponding unsatisfied demands $F(R)$. The maximum unsatisfied demands are total amount of all commodities' demands. The first interdiction budget under which the optimal solution of formulation (8) reaches this maximum value should be $R_{b}$. Additionally, Theorem 5 can be used to obtain the lower bound for critical budget $R_{b}$.

Table 3 presents the results for binary interdiction in 12 networks. The cases that the interdiction budget is close to the critical budget are included (the dots in Table 3 denote those cases we do not include here). We have verified that the critical budgets $R_{b}$ from Theorem 5 is the same as the one obtained by using formulation (8). Actually, in all these cases, the maximum flow obtained from formulation (9) is equal to the minimum disconnect set from formulation (10).

From Table 3, comparing cases with same number of nodes and commodities but different numbers of arcs, we can find that if the number of arcs is reducing, $R_{b}$ will decrease. With less arcs, $R_{b}$ decreases because less arcs need to be removed. On the other hand, we can find cases with same number of nodes and arcs, $R_{b}$ decreases 
Table 3 Critical budgets for binary interdiction

\begin{tabular}{|c|c|c|c|c|c|c|c|c|c|c|c|c|}
\hline \multirow{3}{*}{$\begin{array}{r}|N| \\
|A| \\
K\end{array}$} & \multicolumn{12}{|c|}{8} \\
\hline & \multicolumn{6}{|c|}{30} & \multicolumn{6}{|c|}{40} \\
\hline & \multicolumn{2}{|c|}{3} & \multicolumn{2}{|c|}{4} & \multicolumn{2}{|c|}{6} & \multicolumn{2}{|c|}{3} & \multicolumn{2}{|c|}{4} & \multicolumn{2}{|c|}{6} \\
\hline & $R$ & $F(R)$ & $R$ & $F(R)$ & $R$ & $F(R)$ & $R$ & $F(R)$ & $R$ & $F(R)$ & & $F(R)$ \\
\hline & 10 & 0 & 10 & 0 & 4 & 0 & 21 & 0 & 21 & 0 & 8 & 0 \\
\hline & 11 & 0 & 11 & 0 & 5 & 0 & 22 & 0 & 22 & 0 & 9 & 0 \\
\hline & 12 & 0 & 12 & 0 & 6 & 0 & 23 & 0 & 23 & 0 & 10 & 0 \\
\hline & 13 & 5 & 13 & 5 & 7 & 1 & 24 & 2 & 24 & 6 & 11 & 5 \\
\hline & 14 & 5 & 14 & 5 & 8 & 1 & 25 & 2 & 25 & 6 & 12 & 5 \\
\hline & 15 & 5 & 15 & 5 & 9 & 1 & 26 & 7 & 26 & 7 & 13 & 5 \\
\hline & : & : & : & ${ }^{\circ}$ & : & 0 & & & & & & \\
\hline & 49 & 16 & 55 & 22 & 73 & 35 & 73 & 16 & 84 & 22 & 107 & 37 \\
\hline & 50 & 16 & 56 & 22 & 74 & 35 & 74 & 16 & 85 & 22 & 108 & 37 \\
\hline & 51 & 21 & 57 & 27 & 75 & 40 & 75 & 21 & 86 & 27 & 109 & 40 \\
\hline & 52 & 21 & 58 & 27 & 75 & 40 & 76 & 21 & 87 & 27 & 110 & 40 \\
\hline & 53 & 21 & 59 & 27 & 75 & 40 & 77 & 21 & 88 & 27 & 111 & 40 \\
\hline$R_{a}$ & \multicolumn{2}{|c|}{12} & \multicolumn{2}{|c|}{12} & \multicolumn{2}{|c|}{6} & \multicolumn{2}{|c|}{23} & \multicolumn{2}{|c|}{23} & \multicolumn{2}{|c|}{10} \\
\hline$R_{b}$ & \multicolumn{2}{|c|}{51} & & & \multicolumn{2}{|c|}{75} & & & \multicolumn{2}{|c|}{86} & \multicolumn{2}{|c|}{109} \\
\hline$|N|$ & \multicolumn{6}{|c|}{8} & \multicolumn{6}{|c|}{10} \\
\hline$|A|$ & \multicolumn{6}{|c|}{50} & \multicolumn{6}{|c|}{60} \\
\hline$K$ & & & & & & & & & & & & \\
\hline & $R$ & $F(R)$ & $\bar{R}$ & $F(R)$ & $R$ & $F(R)$ & $R$ & $\overline{F(R)}$ & $R$ & $\overline{F(R)}$ & $R$ & $F(R)$ \\
\hline & 29 & 0 & 29 & 0 & 8 & 0 & 14 & 0 & 14 & 0 & 14 & 0 \\
\hline & 30 & 0 & 30 & 0 & 9 & 0 & 15 & 0 & 15 & 0 & 15 & 0 \\
\hline & 31 & 0 & 31 & 0 & 10 & 0 & 16 & 0 & 16 & 0 & 16 & 0 \\
\hline & 32 & 6 & 32 & 6 & 11 & 5 & 17 & 3 & 17 & 3 & 17 & 3 \\
\hline & 33 & 6 & 33 & 6 & 12 & 5 & 18 & 4 & 18 & 4 & 18 & 4 \\
\hline & 34 & 6 & 34 & 6 & 13 & 5 & 19 & 4 & 19 & 4 & 19 & 4 \\
\hline & $\therefore$ & : & : & : & : & . & : & . & & : & . & : \\
\hline & & . & . & & & & . & & & . & & . \\
\hline & 96 & 17 & 129 & 24 & 162 & 37 & 72 & 18 & 91 & 28 & 121 & 44 \\
\hline & 97 & 17 & 130 & 24 & 163 & 37 & 73 & 18 & 92 & 28 & 122 & 44 \\
\hline & 98 & 17 & 131 & 24 & 164 & 37 & 74 & 19 & 93 & 28 & 123 & 44 \\
\hline & 99 & 20 & 132 & 27 & 165 & 40 & 75 & 23 & 94 & 32 & 124 & 48 \\
\hline & 100 & 20 & 133 & 27 & 166 & 40 & 76 & 23 & 95 & 32 & 125 & 48 \\
\hline & 101 & 20 & 134 & 27 & 167 & 40 & 77 & 23 & 96 & 32 & 126 & 48 \\
\hline$R_{a}$ & & & & & & & & & & & & \\
\hline$R_{b}$ & & & & & & & & & & & & \\
\hline
\end{tabular}

when $K$ decreases. This is because for a same network, number of nodes and arcs that are needed for shipping commodities is reducing as $K$ decreases.

\subsection{Numerical experiment for effects of budget increasing}

In this section, we perform numerical experiment for the effects of budget increasing. We test on network where $|N|=8,|A|=30, K=3$. There is only one destination node for each commodity, and the demand amounts are 5,7,9. The sum set $\mathscr{D}$ as discussed in Section 3.3 is $\mathscr{D}=\{0,5,7,9,12,14,16,21\}$.

For the continuous interdiction case with $p_{i}=1$ for all $i \in N$ and $q_{i j}=1$ for all $(i, j) \in A$ ( $R_{a}=1.2, R_{b}=8$ in this case), we gradually increase budget $R$ from 0 to 9 with step 0.2 and use (8) to calculate the amount of unsatisfied demands. The result for values of $F(R)$ are pictured in Fig. 1(b) in Section 3.1. Theorem 8 for marginal revenue in this test case can be verified. From Fig. 1(a) in Section 3.1, if $F(R) \notin \mathscr{D}$, 
the marginal revenue is positive. Also, the curve $F(R)$ has 7 plateaus, which is less than $|\mathscr{D}|=8$, a conclusion from Corollary 2 .

For the binary interdiction case, we also test the same network with the same values for interdiction costs. Note as values for $p_{i}, q_{i j}$ are integers, we just need to compute $F(R)$ when budget $R$ is an integer (implied by Theorem 9 and Corollary 3 ). Thus, we gradually increase budget from 0 to 9 by step 1 and use formulation (8) to calculate the amount of unsatisfied demands under each budget. The result for values of $F(R)$ is pictured in Fig. 1(a) in Section 3.1.

\section{Conclusions}

In this paper, we have proposed the general interdiction models of the multicommodity flow problem, with consideration of both node and arcs removals and decrease of their capacities. We first proposed reformulation techniques to solve these models in both binary and continuous interdiction cases. Next, we defined the function $F(R)$ as the minimum amount of unsatisfied demands in the resulted network after worst-case interdiction with budget $R$. The function can be obtained by solving a series of similar problems as interdiction models. As a major contribution, we studied the propositions of this functions, including two types of critical budgets, and effects of budget increasing. The analysis of this function will help the interdictor to decide the better use of limited resources for destroying enemy's supplies, controlling of infectious decease, and many other applications. Also, for solving general bilevel programming problems, our proposed information from critical budgets can help to decide when the strong duality property can be applied.

The future research directions include designing efficient algorithm for solving the continuous interdiction case by combining column generation algorithm with our proposed formulation, and studying approaches to find critical budgets in networks with special structures to reduce the computational complexity. Another research direction is to redesign a new network based on the worst interdiction scenarios. Additionally, potential applications can be found for vulnerability analysis of power grids, telecommunication networks, water infrastructures, social networks, etc.

\section{References}

1. Durbin, E.P.: An interdiction model of highway transportation. RM-4945-PR, Rand Corporation, Santa Monica, CA (1966)

2. Wollmer, R.D.: Removing arcs from a network. J. Oper. Res. Soc. AM. 12, 934-940 (1964)

3. Assimakopoulos, N.: A network interdiction model for hospital infection control. Comput. Biol. Med. 17(6), 413-422 (1987)

4. Pulat, H.: A two-sided optimization of border patrol interdiction. Master's Thesis, Naval Postgraduate School, Monterey, CA (2005)

5. Fan, F., Charlton, W., Morton, D.P.: A stochastic program for interdicting smuggled nuclear material. In: Woodruff, D.L. (Ed.), Network Interdiction and Stochastic Integer Programming, Operations Research/Computer Science Interfaces Series. 22, 1-19 (2002)

6. Morton, D.P., Pan, F., Saeger, K.J.: Models for nuclear smuggling interdiction. IIE Trans. 39, 3-14 (2007)

7. Collado, R.A., Papp, D.: Network interdiction - models, applications, unexplored directions. Rutcor Research Report, RRR 4-2012, Rutgers Center for Operations Research, Rutgers University (2012) 
8. Garg, M., Smith, J.C.: Models and algorithms for the design of survivable multicommodity flow networks with general failure scenarios. OMEGA 36, 1057-1071 (2008)

9. Liberatore, F., Scaparra, M.P., Daskin, M.S.: Analysis of facility protection strategies against an uncertain number of attacks: The stochastic R-interdiction median problem with fortification. Comput. Oper. Res. 38(1), 357-366 (2010)

10. Johnston, M., Lee, H., Modiano, E.: A robust optimization approach to backup network design with random failures. Proc. INFOCOM. 1512-1520 (2011)

11. Israeli, E., Wood, R.K.: Shortest-path network interdiction. Networks 40(2), 97-111 (2002)

12. Golden, B.: A problem in network interdiction. Nav. Res. Logist. Q. 25(4), 711-713 (1978)

13. Zenklusen, R.: Matching interdiction. Discrete Appl. Math. 158(15), 1676-1690 (2010)

14. McMasters, A.W., Mustin, T.M.: Optimal interdiction of a supply network. Nav. Res. Logist. Q. 17, 261-268 (1970)

15. Ghare, P.M., Montgomery, D.C., Turner, W. C.: Optimal interdiction policy for a flow network. Nav Res. Logist. Q. 18(1), 37-45 (1971)

16. Cormican, K., Morton, D., Wood, K.: Stochastic network interdiction. Oper. Res. 46, 184-197 (1998)

17. Washburn, A., Wood, R.K.: Two-person zero-sum games for network interdiction. Oper. Res. 43(2), 243-251 (1995)

18. Fulkerson, D.R., Harding, G.C.: Maximizing minimum source-sink path subject to a budget constraint. Math. Program. 13(1), 116-118 (1977)

19. Zheng, Q.P., Shen, S., Shi, Y.: Loss-constrained minimum cost flow under arc failure uncertainty with applications in risk-aware kidney exchange. IIE Trans. 47, 961-977 (2015)

20. Phillips, C.A.: The network inhibition problem. In proceeding of the twenty-fifth annual ACM symposium on Theory of computing. 776-785 (1993)

21. Royset, J. O., Wood, R. K.: Solving the Bi-Objective Maximum-Flow Network-Interdiction Problem. INFORMS J. Comput. 175-184 (2007)

22. Wood, R.K.: Deterministic network interdiction. Math. Comput. Model. 17(2), 1-18 (1993)

23. Atkinson, M.P., Cao, Z., Wein, L.M.: Optimal stopping analysis of a radiation detection system to protect cities from a nuclear terrorist attack. Risk Anal. 28(2), 353-371 (2008)

24. Lim, C., Smith, J.C.: Algorithms for discrete and continuous multicommodity flow network interdiction problems. IIE Trans. 39, 15-26 (2007)

25. Smith, J.C., Lim, C., Sudargho, F.: Survivable network design under optimal and heuristic interdiction scenarios. J. Global. Optim. 38(2), 181-199 (2007)

26. Sullivan, K.M., Smith, J.C.: Exact algorithms for solving a Euclidean maximum flow network interdiction problem. Networks 64(2), 109-124 (2014)

27. Barnhart, C.: Dual-ascent methods for large-scale multicommodity flow problems. Nav. Res. Logist. 40(3), 305-324 (1993)

28. Barnhart, C., Sheffi, Y.: A network-based primal-dual heuristic for the solution of multicommodity network flow problems. Transport. Sci. 27(2), 102-117 (1993)

29. Royset, J.O., Wood, R.K.: Solving the bi-objective maximum-flow network-interdiction problem. INFORMS J. Comput. 19(2),175-184 (2007)

30. Kennington, J.L.: A survey of linear cost multicommodity network flows. Oper. Res. 26(2), 209-236 (1978)

31. Wood, R.K.: Bilevel network interdiction models: Formulations and solutions. Wiley Encyclopedia of Operations Research and Management Science. John Wiley \& Sons, Inc. (2010)

32. Ahuja, R.K., Magnanti, T.L., Orlin, J.B.: Network flows: theory, algorithms, and applications. Prentice Hall, pp. 41-42. Englewood Cliffs, NJ (1993)

33. Aneja, Y.P., Ke, X.: Multicommodity disconnecting set problem. Inter. J.Oper. Res. 4(3), 165-171 (2007)

34. Hu, T. C.: Multi-commodity network flows. Oper. Res. 11(3), 344-360 (1963)

35. Bellmore, M., Greenberg, H.J., Jarvis, J.J.: Multicommodity disconnecting sets. Manage. Sci. 16(6), B-427-B-433 (1970)

36. Dahlhaus, E., Johnson, D.S., Papadimitriou, C.H., Seymour, P.D., Yannakakis, M.: The complexity of multiterminal cuts. Siam. J. Comput. 23(4), 864 - 894 (1994)

37. [URL] http://www.di.unipi.it/optimize/Data/MMCF.html\#NetDesMMCF.

38. Dantzig, G.B.: Upper bounds, secondary constraints, and block triangularity in linear programming. Econometrica, 23(2), 174-183 (1955) 


\section{A Proof of Proposition 1}

Proof. By the construction process of network $G^{\prime}$ from $G$, the objectives in (2) are the same for both networks, as $c_{i_{1}, i_{2}}^{\prime}=0$ for any $i \in N$ and $c_{i_{2}, j_{1}}^{\prime}=c_{i j}$ for any $(i, j) \in A$.

Let $z_{i_{1}, i_{2}}^{\prime k}$ for $i \in A$ and $z_{i_{2}, j_{1}}^{\prime k}$ for $(i, j) \in A$ be commodity flow of type $k \in K$ in network $G^{\prime}$. Similarly, let $y_{i_{1}, i_{2}}^{\prime k}$ for $i \in A$ and $y_{i_{2}, j_{1}}^{\prime k}$ for $(i, j) \in A$ be corresponding interdiction variables on arcs of $G^{\prime}$, and let $\mathbf{y}^{\prime}$ denote the vector of all these interdiction variables, which is limited by $\mathscr{I}$ with the budget $R$ and interdiction costs $q_{i_{1}, i_{2}}^{\prime}, q_{i_{2}, j_{1}}^{\prime}$ are only on arcs. The budget constraint $\sum_{i \in N} p_{i} x_{i}+\sum_{(i, j) \in A} q_{i j} y_{i j} \leq R$ becomes $\sum_{(i, j) \in A^{\prime}} q_{i j}^{\prime} y_{i j}^{\prime} \leq R$.

Constraints (2b) and (2e) are equivalent for both networks $G$ and $G^{\prime}$ (see [32] for explanations). In model (2), except the source and destination nodes, node-capacity constraints $\sum_{j:(i, j) \in A} \sum_{k \in K} z_{i j}^{k} \leq v_{i}(1-$ $\left.x_{i}\right)$ can be equivalently transformed into arc-capacity constraints $\sum_{k \in K} z_{i_{1}, i_{2}}^{\prime k} \leq u_{i_{1}, i_{2}}^{\prime}\left(1-y_{i_{1}, i_{2}}^{\prime}\right)$ in $G^{\prime}$, while constraints $\sum_{k \in K} z_{i j}^{k} \leq u_{i j}\left(1-y_{i j}\right)$ in $G$ become $\sum_{k \in K} z_{i_{2}, j_{1}}^{\prime k} \leq u_{i_{2}, j_{1}}^{\prime}\left(1-y_{i_{2}, j_{1}}^{\prime}\right)$ in $G^{\prime}$. Additionally, nodecapacity constraint $\sum_{j:(i, j) \in A} \sum_{k \in K} z_{i j}^{k} \leq v_{i}\left(1-x_{i}\right)$ for source node $i \in G$ is equivalent to $\sum_{k \in K} z_{i_{1}, i_{2}}^{\prime k} \leq$ $u_{i_{1}, i_{2}}^{\prime}\left(1-y_{i_{1}, i_{2}}^{\prime}\right)$ on arc $\left(i_{1}, i_{2}\right)$ in $G^{\prime}$, where $u_{i_{1}, i_{2}}^{\prime}=v_{i}$. Node-capacity constraint $\sum_{j:(i, j) \in A} \sum_{k \in K} z_{i j}^{k} \leq$ $v_{i}\left(1-x_{i}\right)$ for destination node $i \in G$ now becomes $\sum_{k \in K} z_{i_{1}, i_{2}}^{\prime k} \leq u_{i_{1}, i_{2}}^{\prime}\left(1-y_{i_{1}, i_{2}}^{\prime}\right)$ on arc $\left(i_{1}, i_{2}\right)$ in $G^{\prime}$, where $u_{i_{1}, i_{2}}^{\prime}=v_{i}$. Thus, the node interdiction on $i$ in $G$ is equivalent to the interdiction on $\operatorname{arc}\left(i_{1}, i_{2}\right)$ in $G^{\prime}$, which eventually reduces the out flow of $i_{2}$. For any other $\operatorname{arc}\left(i_{2}, j_{1}\right)$, for any $(i, j) \in A$, in $A^{\prime}$, the arc-capacity constraints in (2d) become $\sum_{k \in K} z_{i j}^{\prime k} \leq u_{i j}^{\prime}\left(1-y_{i j}^{\prime}\right)$. Thus, the arc interdiction on arc $(i, j)$ in $G$ is equivalent to the interdiction on $\operatorname{arc}\left(i_{2}, j_{1}\right)$ in $G^{\prime}$.

Therefore, we have proved the equivalence between model (2) for $G$ with interdiction on both nodes and arcs and model (2) for $G^{\prime}$ with interdiction only on arcs.

\section{B Proof of Theorem 1}

Proof. Formulation (2) can be formulated equivalently as follows:

$$
\begin{aligned}
\max _{\mathbf{x}, \mathbf{y} ; \alpha, \beta, \gamma} & \sum_{k \in K}\left(\sum_{i \in S(k)} s_{i}^{k} \alpha_{i k}-\sum_{i \in D(k)} d_{i}^{k} \alpha_{i k}\right)+\sum_{i \in N} v_{i}\left(1-x_{i}\right) \beta_{i}+\sum_{(i, j) \in A} u_{i j}\left(1-y_{i j}\right) \gamma_{i j} \\
\text { s.t. } & \alpha_{i k}-\alpha_{j k}+\beta_{i}+\gamma_{i j} \leq c_{i j}, \forall k \in K,(i, j) \in A \\
& \beta_{i}, \gamma_{i j} \leq 0, \forall i \in N,(i, j) \in A \\
& \sum_{i \in N} p_{i} x_{i}+\sum_{(i, j) \in A} q_{i j} y_{i j} \leq R \\
& 0 \leq x_{i}, y_{i j} \leq 1, \forall i \in N,(i, j) \in A
\end{aligned}
$$

By the assumption of $R$ such that all demands can be satisfied for any interdiction plans in $\mathscr{I}_{c}$, the inner problem is always feasible. Through strong duality for the multicommodity flow in the resulted network, the above formulation (B.1) under this assumption is equivalent to formulation (2) with $\mathscr{I}=\mathscr{I}_{c}$ for continuous interdiction.

Assume that the set $\mathscr{I}_{c}$ has $L$ extreme points, in the set $\operatorname{ext}\left(\mathscr{I}_{c}\right)=\left\{\left(x^{(1)}, y^{(1)}\right), \cdots,\left(x^{(L)}, y^{(L)}\right)\right\}$. As the constraints of formulation (B.1) can be divided into two parts: constraints (B.1b)-(B.1c) are only related to $\alpha, \beta, \gamma$ while constraints (B.1d)-(B.1e) (i.e., $\mathscr{I}_{c}$ ) are only related to $\mathbf{x}, \mathbf{y}$, the objective function (B.1a) can be reformulated as

$$
\max _{\alpha, \beta, \gamma} \max _{\mathbf{x}, \mathbf{y}} \sum_{k \in K}\left(\sum_{i \in S(k)} s_{i}^{k} \alpha_{i k}-\sum_{i \in D(k)} d_{i}^{k} \alpha_{i k}\right)+\sum_{i \in N} v_{i}\left(1-x_{i}\right) \beta_{i}+\sum_{(i, j) \in A} u_{i j}\left(1-y_{i j}\right) \gamma_{i j}
$$

which is a linear program for any fixed $\alpha, \beta, \gamma$, and the corresponding optimal $\mathbf{x}, \mathbf{y}$ should be obtained at some extreme point of $\mathscr{I}_{c}$, and vice versa. Therefore, (B.2) can be equivalently rewritten by considering constraints in (B.1) as follows:

$$
\max _{\alpha, \beta, \gamma ;(\mathbf{x}, \mathbf{y}) \in \operatorname{ext}\left(\mathscr{I}_{c}\right)} \sum_{k \in K}\left(\sum_{i \in S(k)} s_{i}^{k} \alpha_{i k}-\sum_{i \in D(k)} d_{i}^{k} \alpha_{i k}\right)+\sum_{i \in N} v_{i}\left(1-x_{i}\right) \beta_{i}+\sum_{(i, j) \in A} u_{i j}\left(1-y_{i j}\right) \gamma_{i j}
$$




$$
\begin{array}{ll}
\text { s.t. } & \alpha_{i k}-\alpha_{j k}+\beta_{i}+\gamma_{i j} \leq c_{i j}, \forall k \in K,(i, j) \in A \\
& \beta_{i}, \gamma_{i j} \leq 0, \forall i \in N,(i, j) \in A
\end{array}
$$

As explained in [24] based on the Dantzig's theorem in [38], the following conclusion is used for finding solutions of $\mathbf{x}, \mathbf{y}$ : For each extreme point $\left(\mathbf{x}^{(l)}, \mathbf{y}^{(l)}\right)(l=1, \cdots, L)$ of the polyhedral set $\mathscr{I}_{c}$, there exists a single basic variable in the vector $\left(\mathbf{x}^{(l)}, \mathbf{y}^{(l)}\right)$ taking value in the interval $[0,1]$, while all other variables of $\left(\mathbf{x}^{(l)}, \mathbf{y}^{(l)}\right)$ are nonbasic variables taking values of either 0 or 1.

Therefore, the continuous interdiction model is corresponding to the model where only one node or arc is partially interdicted, the rest interdictions are binary. Let $\delta_{i}, \sigma_{i j} \in\{0,1\}$ associated with $x_{i}, y_{i j}$ denote whether $x_{i}, y_{i j}$ take 1 if $\delta_{i}=1, \sigma_{i j}=1$, or $x_{i}, y_{i j}$ take 0 if $\delta_{i}=0, \sigma_{i j}=0$, respectively. Let $\delta_{i}^{\prime}, \sigma_{i j}^{\prime} \in\{0,1\}$ associated with $x_{i}, y_{i j}$ denote whether $x_{i}, y_{i j}$ take binary values if $\delta_{i}^{\prime}=0, \sigma_{i j}^{\prime}=0$ or $x_{i}, y_{i j}$ take any value in $[0,1]$ if $\delta_{i}^{\prime}=1, \sigma_{i j}^{\prime}=1$, respectively. By the conclusion above, the choices of $\delta_{i}^{\prime}$ 's and $\sigma_{i j}^{\prime}$ 's should satisfy $\sum_{i \in N} \delta_{i}^{\prime}+\sum_{(i, j) \in A} \sigma_{i j}^{\prime}=1$. Therefore, decision variables $\mathbf{x}, \mathbf{y}$ can be replaced by $\delta, \delta^{\prime}, \sigma, \sigma^{\prime}$ (vectors formed correspondingly), and we have the following equivalent formulation for continuous interdiction through the reformulation of (B.3) as follows:

$$
\begin{aligned}
& \max _{\delta, \delta^{\prime}, \sigma, \sigma^{\prime}} \min _{\mathbf{z}} \sum_{(i, j) \in A} c_{i j} \sum_{k \in K} z_{i j}^{k} \\
& \text { s.t. } \text { constraints in (2b) } \\
&\left\{\begin{array}{l}
\sum_{j:(i, j) \in A} \sum_{k} z_{i j}^{k} / v_{i} \leq 1-\delta_{i}-\delta_{i}^{\prime} R^{\prime} / p_{i}, \forall i \in N \\
\sum_{k \in K} z_{i j}^{k} / u_{i j} \leq 1-\sigma_{i j}-\sigma_{i j}^{\prime} R^{\prime} / q_{i j}, \forall(i, j) \in A
\end{array}\right. \\
&\left\{\begin{array}{l}
R^{\prime} / p_{i} \leq 1+M_{i}\left(1-\delta_{i}^{\prime}\right), \forall i \in N \\
R^{\prime} / q_{i j} \leq 1+M_{i j}\left(1-\sigma_{i j}^{\prime}\right), \forall(i, j) \in A
\end{array}\right. \\
& \sum_{i \in N} p_{i} \delta_{i}+\sum_{(i, j) \in A} q_{i j} \sigma_{i j}+R^{\prime}=R \\
& \delta_{i}+\delta_{i}^{\prime} \leq 1, \forall i \in N \\
& \sigma_{i j}+\sigma_{i j}^{\prime} \leq 1, \forall(i, j) \in A \\
& \sum_{i \in N} \delta_{i}^{\prime}+\sum_{(i, j) \in A} \sigma_{i j}^{\prime}=1 \\
& \delta_{i}, \sigma_{i j}, \delta_{i}^{\prime}, \sigma_{i j}^{\prime} \in\{0,1\}, \forall i \in N,(i, j) \in A \\
& R^{\prime} \geq 0 ; z_{i j}^{k} \geq 0, \forall(i, j) \in A, k \in K
\end{aligned}
$$

The interdiction constraint (B.4e) limits the budget for all binary interdictions with constraint $R^{\prime} \geq 0$, where $R^{\prime}=R-\left(\sum_{i \in N} p_{i} \delta_{i}+\sum_{(i, j) \in A} q_{i j} \sigma_{i j}\right)$ is the remaining budget for a partial disruption of a node or an arc. Constraints (B.4f)-(B.4g) indicate that a node and an arc can be either binary interdicted or partially interdicted or not interdicted, respectively. Constraint (B.4h) limits that only one node or one arc is partially interdicted. The first set of constraints in (B.4c) include cases for node interdiction: (i) $\delta_{i}=1, \delta_{i}^{\prime}=0$, node $i$ is completely interdicted; (ii) $\delta_{i}=0, \delta_{i}^{\prime}=0$, node $i$ is not interdicted, and this set of constraints limits the node capacity; (iii) $\delta_{i}=0, \delta_{i}^{\prime}=1$, node $i$ is partially interdicted, and this set of constraints limits the capacity after partially interdicted. Similarly, the second set of constraints in (B.4c) are for arc interdiction. The parameters $M_{i}$ and $M_{i j}$ in (B.4d) are relatively large positive constants. The first set of constraints in (B.4d) ensures that the budget spent on partially interdicted node should be limited by corresponding node interdiction cost, while second set limits the amount for budget spent on partially interdicted arc. The objective function and all other constraints have the same meanings as those in formulation (2).

Let $\alpha_{i k}, \beta_{i}, \gamma_{i j}$ be the dual variables associated with constraints in (B.4b)-(B.4c), under certain $R$, the formulation (B.4) is equivalent to :

$$
\begin{aligned}
& \max _{\delta, \delta^{\prime}, \sigma, \sigma^{\prime}, \alpha, \beta, \gamma} \sum_{k \in K}\left(\sum_{i \in O(k)} s_{i}^{k} \alpha_{i k}-\sum_{i \in D(k)} d_{i}^{k} \alpha_{i k}\right) \\
&+\sum_{i \in N} \beta_{i}\left(1-\delta_{i}-\delta_{i}^{\prime} R^{\prime} / p_{i}\right)+\sum_{(i, j) \in A} \gamma_{i j}\left(1-\sigma_{i j}-\sigma_{i j}^{\prime} R^{\prime} / q_{i j}\right) \\
& \text { s.t. } \alpha_{i k}-\alpha_{j k}+\beta_{i} / v_{i}+\gamma_{i j} / u_{i j} \leq c_{i j}, \forall(i, j) \in A, \forall k \in K
\end{aligned}
$$


constraints in (B.4d) - (B.4i)

(B.5c)

$R^{\prime} \geq 0 ; \beta_{i}, \gamma_{i j} \leq 0, \forall(i, j) \in A, \forall k \in K$

This completes the proof. 